\title{
Spectral Analysis of $N$-Body Stark Hamiltonians
}

\author{
Ira Herbst ${ }^{1, \star}$, Jacob Schach Møller ${ }^{2}$, Erik Skibsted ${ }^{2}$ \\ 1 Department of Mathematics, University of Virginia, Charlottesville, VA 22903, USA \\ 2 Matematisk Institut, Aarhus Universitet, Ny Munkegade, DK-8000 Aarhus C, Denmark
}

Received: 29 August 1994

\begin{abstract}
We prove that the spectrum for a large class of $N$-body Stark Hamiltonians is purely absolutely continuous. We need slow decay at infinity and local singularities of at most Coulomb type. In particular our results include the usual models for atoms and molecules.
\end{abstract}

\section{Section 1. Introduction}

In this paper we prove absence of pure point and singular continuous spectra for a large class of $N$-body Stark Hamiltonians. The model is the following. We consider a system of $N v$-dimensional particles labelled $1, \ldots, N$ with masses, charges, positions and momenta denoted by $m_{l}, q_{l}, x_{i}$, and $p_{l}$, respectively. The interaction consists of two parts. One part (the external part) is due to the presence of an electric field $\mathscr{E}$, while the other part (the internal interaction) is given as a sum of pair potentials each one, $v_{l \jmath}$, assumed to obey the (weak) decay condition,

$$
\left|v_{l j}(y)\right|+\left|\nabla v_{\imath j}(y)\right|=o(1) \text { for } \quad y \rightarrow \infty .
$$

Here and in the first part of this introduction we shall assume $v_{l}$ to be $C^{1}$. We defer the discussion of adding local singularities to the last part of the introduction. The total Hamiltonian reads

$$
\tilde{H}=\sum_{l=1}^{N}\left(\frac{p_{i}^{2}}{2 m_{l}}-q_{l} \mathscr{E} \cdot x_{i}\right)+\sum_{1 \leqq i<j \leqq N} v_{l j}\left(x_{l}-x_{J}\right) .
$$

After the (standard) procedure of separating out the center of mass motion for $\tilde{H}$ we obtain a Hamiltonian $H$ on $L^{2}(X)$, where

$$
X=\left\{x=\left(x_{1}, \ldots, x_{N}\right) \mid x_{l} \in \mathbf{R}^{\prime \prime}, \sum_{l=1}^{N} m_{i} x_{l}=0\right\} .
$$

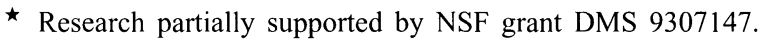


There are two main results in this paper proved under the condition (1.1) and the assumption that $\frac{q_{l}}{m_{i}} \neq \frac{q_{j}}{m_{j}}$ for some pair $i, j \leqq N$. The first is

$$
\sigma_{p p}(H)=\emptyset \text {; }
$$

i.e. that the pure point spectrum of $H$ is empty. For the second one we need an additional assumption on the second order derivatives. The statement is then

$$
\sigma_{s c}(H)=\emptyset
$$

i.e. that the singular continuous spectrum of $H$ is empty. The proof of (1.3) involves resolvent estimates of independent interest (in fact useful in [HMS2]). We obtain globally uniform estimates.

The results above generalize some recent results [Sk, T1-3] obtained in the case of $v_{i j}$ in $C^{1}$. The first of these papers needed an additional geometric condition that was verified for $N=3$ and for "atoms" for $N>3$. The others all needed a stronger condition than (1.1), namely the short range condition given by replacing the righthand side by $O\left(|y|^{-\frac{1}{2}-\varepsilon}\right)$. Previous to the above works is [Si] that contains (1.2) for Born Oppenheimer molecules. Here the condition of repulsion (between electrons) and spherical symmetry was important in the proof.

As was the case for the above mentioned works we shall in this paper apply commutator methods. We adapt the variant of the method of Froese and Herbst $[\mathrm{FH}]$ used in [Sk]. The main difference between [Sk, T3] and our paper is that in a certain inductive step one needs a certain statement for subsystems to be uniform with respect to translations of the energy. While the other papers dealt only with translations to the left (which is easier and in some sense elementary) we overcome in this paper the problem of proving uniformity with respect to all translations (cf. [T1-2] for the case $N=3$ ). For that a result proved previously by one of the authors [H1, Proposition 2.1] is very convenient. Once this point is circumvented the somewhat sophisticated vector fields of [Sk] and [T3] are not needed. As a matter of fact the vector field we use for proving (1.2) is the (contravariant) gradient field corresponding to the function

$$
f(x)=\kappa\left(1+\sum_{i=1}^{N} 2 m_{l}\left|x_{l}\right|^{2}\right)^{\frac{1}{2}}+\sum_{i=1}^{N} q_{l} \mathscr{E} \cdot x_{l} ; \quad \kappa \gg 1 .
$$

The one we use for proving (1.3) is even simpler, namely the gradient field corresponding to the function given by the second term on the right-hand side of (1.4). We apply the Mourre method [M] to show (1.3).

As for local singularities we can include singularities of Coulomb type; for example if we add to the previous type of pair potential (1.1) a potential of the form

$$
v_{l j}=q_{\imath} q_{j}\left|x_{i}-x_{j}\right|^{-1} ; \quad v \geqq 3,
$$

then (1.2) and (1.3) remain valid. In addition we can treat sums of Coulomb potentials with different centers of the singularities and in fact our results include the well known Born Oppenheimer model for molecules with fixed nuclei. It should be noticed that this kind of singularity is a borderline case from the point of view of our methods. For example they assure relative boundedness while for slightly stronger singularities this property does not hold (in $N$-body Stark problems). The basic 
schemes for the proofs in the case of singularities are the same as before, however there are some additional technical difficulties, for example while (1.4) can be used again (for (1.2)) the simple vector field used for (1.3) does not work in the general case (because of some troubles with the double commutator in the Mourre theory). The latter problem is circumvented by introducing a more complicated vector field that can be viewed as a modification of the vector field constructed in [T3].

We shall use the framework of generalized Schrödinger operators. Our results (in the $C^{1}$ case) are presented in this framework in Sect. 2. The next section contains preliminary results. The crucial uniform statement referred to above is formulated as Lemma 3.6. A more general result of independent interest is Proposition 3.7. In Sect. 4 we prove various estimates that we use to prove the result corresponding to (1.2). In Sect. 5 we shall outline a proof of the result corresponding to (1.3) and of some resolvent estimates. In Sect. 6 we discuss how to include local singularities. For this purpose we have added two appendices. The first, Appendix A, concerns the proof of Proposition 6.4 from which in particular we can infer that the Coulomb singularity is the borderline case for relative boundedness (cf. the discussion above). In Appendix B we construct the vector field mentioned above to obtain (1.3) and resolvent estimates in the general case.

This paper is naturally viewed as the first in a series of two. Our second paper [HMS2] will deal with asymptotic completeness for $N$-body Stark Hamiltonians.

\section{Section 2. Notations, Assumptions and Results}

We consider a finite dimensional real vector space $X$ with an inner product $x \cdot y$, and a finite family $\left\{X_{a} \mid a \in \mathscr{A}\right\}$ of subspaces of $X$. It is assumed that this family is closed with respect to intersection and that there exist $a_{\min }, a_{\max } \in \mathscr{A}$ such that $X_{a_{\mathrm{min}}}=X$ and $X_{a_{\max }}=0$. The orthogonal complement of $X_{a}$ in $X$ is denoted by $X^{a}$. Clearly we can assume that $X_{a}=X_{b} \Rightarrow a=b$. Then we can order $\mathscr{A}$ by writing $a_{1} \subset a_{2}$ if $X^{a_{1}} \subset X^{a_{2}}$. For given $a$ we define $\# a$ as the largest $n \in \mathbf{N}$ for which there exists an increasing sequence $a=a_{n} \subsetneq a_{n-1} \subsetneq \cdots \subsetneq a_{1}=a_{\max }$. The largest such $n$ is $N:=\# a_{\min }$. The orthogonal projections onto $X_{a}$ and $X^{a}$ are denoted by $\Pi_{a}$ and $\Pi^{a}$, respectively. The corresponding components for $x \in X$ are denoted $x_{a}$ and $x^{a}$. We use similar notations for the components of the momentum operator $p=-i \nabla=\left(p_{1}, \ldots, p \operatorname{dim} X\right)$. We put $\langle x\rangle=\left(1+|x|^{2}\right)^{\frac{1}{2}} ;|x|^{2}=x \cdot x$. The notations $\langle p\rangle$ and $\langle\lambda\rangle$ for $\lambda \in \mathbf{R}$ are (formally) given by the same expression.

Let $-\Delta=p^{2}$ and $E \in X$. Then the Hamiltonian

$$
H=-\Delta-E \cdot x+V ; \quad V=\sum_{a \in \mathscr{C}} V_{a},
$$

is essentially selfadjoint on $C_{0}^{\infty}(X) \subset L^{2}(X)$ under Condition 2.1 (1) below.

\section{Conditions 2.1.}

(1) For all $a \in \mathscr{A}$ the real potential $V_{a}\left(x^{a}\right) \in C^{1}\left(X^{a}\right)$ obeys

$$
\partial^{\beta} V_{a}\left(x^{a}\right)=o(1) \text { for } x^{a} \rightarrow \infty \text { and }|\beta| \leqq 1 .
$$

(2) For all $a \in \mathscr{A}$ the potential $V_{a}\left(x^{a}\right)$ admits bounded second order distributional derivatives.

By convention $\partial^{\beta} V_{a_{\mathrm{min}}}=0$.

Our main results are 
Theorem 2.2. Suppose Condition 2.1 (1) and $E \neq 0$. Then the pure point spectrum $\sigma_{p p}(H)$ is empty.

Theorem 2.3. Suppose Conditions 2.1 (1), (2) and $E \neq 0$. Then

(1) $\forall \delta>\frac{1}{2}: \sup _{\operatorname{Im} z \neq 0}\left\|\langle p\rangle^{-\delta}(H-z)^{-1}\langle p\rangle^{-\delta}\right\|<\infty$,

(2) $\forall \delta^{\prime}>\frac{1}{4}$ :

$$
\sup _{\operatorname{Im} z \neq 0, \operatorname{Re} z \leqq 0}\langle\operatorname{Re} z\rangle^{v}\left\|\langle x\rangle^{-\delta^{\prime}}(H-z)^{-1}\langle x\rangle^{-\delta^{\prime}}\right\|<\infty ; \quad v<\frac{4 \delta^{\prime}-1}{4 \delta^{\prime}+1}
$$

while

$$
\sup _{\operatorname{Im} z \neq 0, \operatorname{Re} z \geqq 0}\langle\operatorname{Re} z\rangle^{v}\left\|\langle x\rangle^{-\delta^{\prime}}(H-z)^{-1}\langle x\rangle^{-\delta^{\prime}}\right\|<\infty ; \quad v<-\frac{1}{2} .
$$

In particular the singular continuous spectrum $\sigma_{s c}(H)$ is empty. Moreover in both cases the (two) boundary values

$$
\lim _{\operatorname{Im} z \downarrow(\uparrow) 0}\langle p\rangle^{-\delta}(H-z)^{-1}\langle p\rangle^{-\delta}, \quad \lim _{\operatorname{Im} z \downarrow(\uparrow) 0}\langle x\rangle^{-\delta^{\prime}}(H-z)^{-1}\langle x\rangle^{-\delta^{\prime}}
$$

exist in $\mathscr{B}\left(L^{2}(X)\right)$ and are attained uniformly for $\operatorname{Re} z$ in compact sets.

We complete this section with some notations.

For $a \neq a_{\min }$ we introduce the sub-Hamiltonian

and

$$
H^{a}=\left(p^{a}\right)^{2}-E^{a} \cdot x^{a}+V^{a} ; \quad V^{a}=\sum_{b \subset a} V_{b},
$$

$$
H_{a}=p^{2}-E \cdot x+V^{a}=\left(p_{a}\right)^{2}-E_{a} \cdot x_{a}+H^{a} .
$$

Let

$$
H_{0}=p^{2}-E \cdot x \text {. }
$$

For $R>0$ the notation $F(\cdot<R)$ stands for the characteristic function of the interval $(-\infty, R)$. The notation $\chi(\cdot<R)$ stands for a smooth function $\chi: \mathbf{R} \rightarrow[0,1]$ obeying $\chi(t<R)=1$ for $t \leqq R$ and $\chi(t<R)=0$ for $t>2 R$. Let $F(\cdot \geqq R)=1-F(\cdot<R)$ and $\chi(\cdot>R)=1-\chi(\cdot<R)$.

For $\varepsilon>0$ the notation $\eta_{\varepsilon}$ stands for any function $\eta \in C_{0}^{\infty}(\mathbf{R})$ obeying $0 \leqq$ $\eta \leqq 1$ and $\eta(t)=1$ for $|t| \leqq \varepsilon$ and $\eta(t)=0$ for $|t|>2 \varepsilon$.

\section{Section 3. Preliminaries}

In this section we state various basic facts and prepare for an inductive proof of Theorem 2.2 to be given in Sect. 4. All statements hold under Assumption 2.1 (1) and for any fixed $E \in X$.

Lemma 3.1. The domain $\mathscr{D}(H) \subseteq H_{\mathrm{loc}}^{2}$, the local Sobolev space of order 2. Moreover

are bounded.

$$
p\langle x\rangle^{-\frac{1}{2}}(H-i)^{-1} \text { and } p^{2}\langle x\rangle^{-1}(H-i)^{-1}
$$

Proof. By the well known formula

$$
h p^{2} h=\operatorname{Re}\left(h^{2} p^{2}\right)+|\nabla h|^{2}
$$


applied to $h=\langle x\rangle^{-\frac{1}{2}}$ we obtain for $\psi \in L^{2}(X)$ and $\phi=(H-i)^{-1} \psi$ that

$$
\|p h \phi\|^{2}=\left\langle\phi,\left\{\operatorname{Re}\left(h^{2} H\right)+h^{2}(E \cdot x-V)+|\nabla h|^{2}\right\} \phi\right\rangle .
$$

Clearly

$$
\begin{gathered}
\left\langle\phi, \operatorname{Re}\left(h^{2} H\right) \phi\right\rangle=\operatorname{Re}\left\langle\phi, h^{2}(H-i) \phi\right\rangle, \\
\left\|(H-i)^{-1}\right\| \leqq 1,
\end{gathered}
$$

and for some $C>0$,

$$
\left|\left\langle\phi,\left\{h^{2}(E \cdot x-V)+|\nabla h|^{2}\right\} \phi\right\rangle\right| \leqq C\|\phi\|^{2} .
$$

Hence

$$
\|p h \phi\|^{2} \leqq\|\phi\|\|\psi\|+C\|\phi\|^{2} \leqq(1+C)\|\psi\|^{2},
$$

which proves boundedness of the first operator.

As for the boundedness of the second operator it follows readily from the result proved above.

By a similar method one can prove

Lemma 3.2. Suppose $0 \leqq h \in C^{\infty}(X)$ and $\psi \in \mathscr{D}(H)$ are given such that $h H \psi, h \psi$, $E \cdot x h \psi,\left(\partial_{j} h\right) \psi \in L^{2}(X)$ for any component $\partial_{j} h$ of $\nabla h$. Then

$$
p_{j} h \psi, h p_{j} \psi \in L^{2}(X) \text {. }
$$

Lemma 3.3. Let $R>0$ and $\mu \in \mathbf{R} \backslash 0$. Then

$$
\left\|F(|x|<R)\left(H_{0}-\lambda-i \mu\right)^{-1} F(|x|<R)\right\| \rightarrow 0 \quad \text { for } \quad|\lambda| \rightarrow \infty .
$$

Proof. It suffices to look at the case $\mu>0$ only. By the formula

$$
\left(H_{0}-\lambda-i \mu\right)^{-1}=i \int_{0}^{\infty} e^{i t(\lambda+i \mu)} e^{-i t H_{0}} d t
$$

it is enough to show that for any $\varepsilon>0$,

$$
\left\|\int_{\varepsilon}^{\infty} F(|x|<R) e^{\imath t(\hat{\lambda}+\imath \mu)} e^{-\imath t H_{0}} F(|x|<R) d t\right\| \rightarrow 0 \quad \text { for } \quad|\lambda| \rightarrow \infty .
$$

For that we use the formula (cf. $[\mathrm{H} 1,(3.10)]$ )

$$
e^{-t t H_{0}}=e^{-l \frac{|E|^{2}}{12} t^{3}} e^{i \frac{t}{2} E \cdot x} e^{-i t p^{2}} e^{i \frac{t}{2} E \cdot x}
$$

and the well known expression

$$
K\left(x, x^{\prime}\right)=(4 \pi i t)^{-\frac{\mathrm{d} m X}{2}} e^{i \frac{\left|r-x^{\prime}\right|^{2}}{4 t}}
$$

for the kernel of $e^{-t t p^{2}}$. We write $e^{i t \lambda}=-\frac{i}{\lambda} \frac{d}{d t} e^{i t \lambda}$ and integrate by parts to obtain the bound $O\left(|\lambda|^{-1}\right)$. 
In order to control the high energy behaviour (cf. Lemma 3.6) we also need another bound for the free resolvent. It follows readily from a result of one of the authors [H1, Proposition 2.1] by a simple interpolation.

Lemma 3.4. Suppose $0<\gamma_{1}<\gamma_{2} \leqq \frac{1}{2}, \mu \in \mathbf{R} \backslash 0$ and $\Pi: X \rightarrow X$ is an orthogonal projection. Then

$$
\left\|\langle\Pi x\rangle^{\gamma_{1}}\left(H_{0}-\lambda-i \mu\right)^{-1}\langle\Pi x\rangle^{-\gamma_{2}}\right\| \leqq C,
$$

uniformly with respect to $\lambda \in \mathbf{R}$.

Let $\left\{j_{a}\right\}, a \neq a_{\max }$, be a family of functions on $X$ each one being smooth and homogeneous of degree 0 outside a compact set. Moreover we assume that

$$
\sum_{a} j_{a}(x)=1 \text { for }|x| \text { large }
$$

for any $b \not \subset a$

$$
\frac{\langle x\rangle}{\left\langle x^{b}\right\rangle} \text { is bounded on } \operatorname{supp} j_{a}
$$

and (hence in particular) for any $R>0$ and $b \not \subset a$,

$$
F\left(\left|x^{b}\right|<R\right) j_{a}=0 \text { for }|x| \text { large . }
$$

Lemma 3.5. Let $\varepsilon>0$. Then

(1) $\left\|\eta_{\varepsilon}(H-\lambda) F(|X|<R)\right\| \rightarrow 0$ for $|\lambda| \rightarrow \infty ; R>0$,

(2) $\left\|F(|x| \geqq R)\left\{\eta_{\varepsilon}(H-\lambda)-\eta_{\varepsilon}\left(H_{a}-\lambda\right)\right\} j_{a}\right\| \rightarrow 0$ for $R \rightarrow \infty$,

uniformly with respect to $\lambda \in \mathbf{R} ; a \neq a_{\max }$.

Proof. We pick

$$
\mu>\sum_{a \in \mathscr{C}} \sup \left|V_{a}(x)\right|
$$

Then to show the first statement it suffices, by multiplying by the adjoint and applying the Stone Weierstrass theorem, to prove that

$$
\begin{gathered}
\left\|F(|x|<R)\left(H-\lambda-i \mu_{1}\right)^{-1} \cdots\left(H-\lambda-i \mu_{n}\right)^{-1} F(|x|<R)\right\| \rightarrow 0 \text { for }|\lambda| \rightarrow \infty ; \\
\left|\mu_{j}\right|=\mu \text { for } j \leqq n \in \mathbf{N} .
\end{gathered}
$$

For that we recall the Neumann series

$$
\left(H-\lambda-i \mu_{J}\right)^{-1}=\left(H_{0}-\lambda-i \mu_{j}\right)^{-1} \sum_{m=0}^{\infty}\left\{-V\left(H_{0}-\lambda-i \mu_{j}\right)^{-1}\right\}^{m},
$$

which is convergent by (3.5). By truncating this series for each $j$ and inserting into (3.6) we can reduce the proof to a similar statement with $H$ replaced by $H_{0}$ and with bounded functions between the (free) resolvents. For notational convenience we look at the statement (3.6) with $H$ replaced by $H_{0}$ as the only modification. With this change the proof is completed as follows. We pick $0<\gamma_{1}<\cdots<\gamma_{n}=\frac{1}{2}$, insert successively $1=\langle x\rangle^{-\gamma_{j}}\langle x\rangle^{\gamma_{J}}$ and apply Lemma 3.4 to obtain that it suffices to show

$$
\left\|F(|x|<R)\left(H_{0}-\lambda-i \mu_{1}\right)^{-1}\langle x\rangle^{-\gamma_{1}}\right\| \rightarrow 0 \text { for }|\lambda| \rightarrow \infty .
$$


But (3.8) follows from Lemma 3.3 by inserting on the right-hand side

$$
1=F\left(|x|<R^{\prime}\right)+F\left(|x| \geqq R^{\prime}\right)
$$

and choosing $R^{\prime}$ large.

To show the second statement we proceed similarly. By using (3.5) and (3.7) it suffices to prove that for any $b \not \subset a$ and finite products $B_{l}(\lambda), l=1,2$, of bounded functions and free resolvents of the form $\left(H_{0}-\lambda-i \mu_{j}\right)^{-1}$,

$$
\begin{gathered}
\left\|F(|x| \geqq R) B_{1}(\lambda) V_{b} B_{2}(\lambda) j_{a}\right\| \rightarrow 0 \text { for } R \rightarrow \infty, \\
\text { uniformly in } \lambda \in \mathbf{R} .
\end{gathered}
$$

For that we can assume $V_{b}$ to be compactly supported. Then we insert (3.9) to the right and replace $j_{a}$ by $\left(\frac{\left\langle x^{b}\right\rangle}{\langle x\rangle}\right)^{\frac{1}{4}} j_{a}$, the latter justified by (3.3). As a result we need to estimate

$$
A_{1}(\lambda, R)=F(|x| \geqq R) B_{1}(\lambda) V_{b} B_{2}(\lambda)\left(\frac{\left\langle x^{b}\right\rangle}{\langle x\rangle}\right)^{\frac{1}{4}} j_{a} F\left(|x| \geqq R^{\prime}\right)
$$

and

$$
A_{2}(\lambda, R)=F(|x| \geqq R) B_{1}(\hat{\lambda}) V_{b} B_{2}(\lambda) j_{a} F\left(|x|<R^{\prime}\right) .
$$

By a similar application of Lemma 3.4 as above we can show that $V_{b} B_{2}(\lambda)\left\langle x^{b}\right\rangle^{\frac{1}{4}}$ is bounded uniformly in $\lambda \in \mathbf{R}$. Hence $A_{1}(\lambda, R)=O\left(R^{\prime-\frac{1}{4}}\right)$ uniformly in $\lambda$ and $R$. We fix $R^{\prime}$ large. Then by the same argument we can write $A_{2}(\lambda, R)=F(|x| \geqq$ $R)\langle x\rangle^{-\frac{1}{4}} A_{3}(\lambda) ; A_{3}(\lambda)$ bounded uniformly in $\lambda$. So clearly $A_{2}(\lambda, R)=O\left(R^{-\frac{1}{4}}\right)$ uniformly in $\lambda$, which completes the proof of (3.10).

Lemma 3.6. Suppose $R>0, a_{\mathrm{m} ! n} \neq b \subset d$ and $\sigma_{p p}\left(H^{a}\right)=\emptyset$ for all $b \subset a \subset d$. Then

$$
\left\|\eta_{\varepsilon}\left(H^{d}-\lambda\right) F\left(\left|x^{b}\right|<R\right)\right\| \rightarrow 0 \text { for } \varepsilon \rightarrow 0,
$$

uniformly in $\lambda \in \mathbf{R}$.

Proof. The proof is by induction in $\# d$ starting from above with $\# d=N-1$, in which case the statement follows from Lemma 3.5 (1) (with $H$ replaced by $H^{d}$ ) and a compactness argument (see the first step of the proof below for details). For notational convenience we shall assume the statement for $\# d>1$ and look at the case $d=a_{\max }$ under this hypothesis.

Suppose first that also $b=a_{\max }$. Then clearly

$$
\left\|\eta_{\varepsilon}(H-\lambda) F(|x|<R)\right\| \leqq\left\|\eta_{1}(H-\lambda) F(|x|<R)\right\|
$$

holds for all $\frac{1}{2}>\varepsilon>0$ and $\lambda \in \mathbf{R}$. By Lemma 3.5 (1) (applied with $\varepsilon=1$ ) the right-hand side goes to zero for $|\lambda| \rightarrow \infty$. Thus it suffices to look at $\lambda$ in a fixed compact set. But the convergence statement of the lemma holds uniformly with respect to such $\lambda$ by a compactness argument since by assumption $\sigma_{p p}(H)=\emptyset$.

To treat a general $b$ as in the lemma we use the partition of unity introduced above. By the statement proved for $b=a_{\max }$ and (3.2), (3.4) it suffices to prove that for any $a \neq a_{\max }$ with $b \subset a$

$$
\left\|\eta_{\varepsilon}(H-\lambda) j_{a} F\left(\left|x^{b}\right|<R\right)\right\| \rightarrow 0 \quad \text { for } \quad \varepsilon \rightarrow 0,
$$


the statement being uniform in $\lambda$. For that we insert

$$
\begin{aligned}
\eta_{\varepsilon}(H-\lambda) j_{a}= & \eta_{\varepsilon}(H-\lambda)\left\{\eta_{\varepsilon^{\prime}}(H-\lambda)-\eta_{\varepsilon^{\prime}}\left(H_{a}-\lambda\right)\right\} j_{a} \\
& +\eta_{\varepsilon}(H-\lambda) \eta_{\varepsilon^{\prime}}\left(H_{a}-\lambda\right) j_{a} ; \quad \varepsilon^{\prime} \geqq 2 \varepsilon .
\end{aligned}
$$

As for the contribution from the second term on the right-hand side we use the fact that

$$
\left\|\eta_{\varepsilon^{\prime}}\left(H_{a}-\lambda\right) F\left(\left|x^{b}\right|<R\right)\right\| \leqq \sup _{v \in \mathbf{R}}\left\|\eta_{\varepsilon^{\prime}}\left(H^{a}+v-\lambda\right) F\left(\left|x^{b}\right|<R\right)\right\|
$$

By the induction hypothesis

$$
\left\|\eta_{\varepsilon^{\prime}}\left(H^{a}-\tilde{\lambda}\right) F\left(\left|x^{b}\right|<R\right)\right\| \rightarrow 0 \quad \text { for } \quad \varepsilon^{\prime} \rightarrow 0,
$$

uniformly in $\tilde{\lambda}=\lambda-v \in \mathbf{R}$. We fix $\varepsilon^{\prime}$ small accordingly to control the contribution from the second term on the right-hand side of (3.11). The contribution from the first term can be dealt with as follows. We need to estimate (cf. (3.9))

$$
A_{1}(\lambda, \varepsilon)=\eta_{\varepsilon}(H-\lambda) F\left(|x| \geqq R^{\prime}\right)\left\{\eta_{\varepsilon^{\prime}}(H-\lambda)-\eta_{\varepsilon^{\prime}}\left(H_{a}-\lambda\right)\right\} j_{a} F\left(\left|x^{b}\right|<R\right)
$$

and

$$
A_{2}(\lambda, \varepsilon)=\eta_{\varepsilon}(H-\lambda) F\left(|x|<R^{\prime}\right)\left\{\eta_{\varepsilon^{\prime}}(H-\lambda)-\eta_{\varepsilon^{\prime}}\left(H_{a}-\lambda\right)\right\}_{j_{a}} F\left(\left|x^{b}\right|<R\right) .
$$

By Lemma $3.5(2)$ the norm of $A_{1}(\lambda, \varepsilon)$ is small for $R^{\prime}$ large uniformly in $\varepsilon$ and $\lambda$. On the other hand (for fixed $R^{\prime}$ )

$$
\left\|\eta_{\varepsilon}(H-\lambda) F\left(|x|<R^{\prime}\right)\right\| \rightarrow 0 \text { for } \varepsilon \rightarrow 0,
$$

uniformly in $\lambda$, as it follows from the first step of the proof above. Hence the norm of $A_{2}(\lambda, \varepsilon)$ is small for $\varepsilon$ small uniformly in $\lambda$.

There is a slight generalization of Lemma 3.6 that we consider of independent interest. Moreover it plays an important role in [HMS2]. Roughly the statement says that shrinking in energy (in fact uniformly with respect to the center of localization) implies spreading with respect to any coordinates not orthogonal to the direction of the field. We anticipate Theorem 2.2 in its formulation.

Proposition 3.7. Suppose $R>0$ and $\Pi: X \rightarrow X$ is an orthogonal projection such that $\Pi E \neq 0$. Then

$$
\left\|\eta_{\varepsilon}(H-\lambda) F(|\Pi x|<R)\right\| \rightarrow 0 \quad \text { for } \quad \varepsilon \rightarrow 0,
$$

uniformly in $\lambda \in \mathbf{R}$.

Proof. We add the projection $I-\Pi$ to the family of projections $\Pi_{a}, a \in \mathscr{A}$. Possibly after another extension we obtain a new family of projections whose ranges fulfill the requirements imposed in the beginning of Sect. 2. Hence the result follows from Lemma 3.6 and Theorem 2.2.

\section{Section 4. Estimates}

In this section we prove Theorem 2.2. This is done by showing by induction in $\# a$ that $\sigma_{p p}\left(H^{a}\right)=\emptyset$ under the assumption that $E^{a} \neq 0$ starting from above with 
$\# a=N-1$, in which case the statement can easily be extracted from the proof below. For notational convenience we shall assume the statement for $\# a>1$ and look at the case $a=a_{\max }$ under this hypothesis.

We shall use a variant of the method of [FH] (see also [AHS] and [H2]), which basically relies on the following computations.

For some smooth functions $\theta: \mathbf{R}_{+} \rightarrow \mathbf{R}$ and $f: X \rightarrow \mathbf{R}_{+}$to be specified below we put

$$
\begin{aligned}
A_{f} & =(\nabla f \cdot p+p \cdot \nabla f) / 2, \\
\theta=\theta(f), \quad \theta^{\prime} & =\left(\frac{d}{d t} \theta\right)(f), \quad \theta^{\prime \prime}=\left(\frac{d^{2}}{d t^{2}} \theta\right)(f),
\end{aligned}
$$

and compute the commutator

$$
\begin{aligned}
i\left[H, e^{\theta} A_{f} e^{\theta}\right] & =e^{\theta}\left\{T_{1}+\cdots+T_{4}\right\} e^{\theta} ; \\
T_{1} & =4 A_{f} \theta^{\prime} A_{f}, \\
T_{2} & =2 p f^{(2)} p, \\
T_{3} & =(E-\nabla V) \cdot \nabla f, \\
T_{4} & =-\frac{1}{2} \Delta^{2} f+\nabla f \cdot \nabla\left(\left(\theta^{\prime 2}-\theta^{\prime \prime}\right)|\nabla f|^{2}\right) .
\end{aligned}
$$

Moreover

$$
\left(H-\lambda-\left(\theta^{\prime 2}-\theta^{\prime \prime}\right)|\nabla f|^{2}+2 i \theta^{\prime} A_{f}\right) e^{\theta}=e^{\theta}(H-\lambda) .
$$

We shall use these formulas with

$$
f(x)=f_{h}(x)=\kappa\langle x\rangle+E \cdot x,
$$

where $\kappa \gg 1$.

As for $\theta$ we have

$$
\frac{d}{d t} \theta(t) \geqq 0, \quad\left(\frac{d^{n}}{d t^{n}}\right) \theta(t)=O\left(t^{1-n}\right) \quad \text { for } \quad t \rightarrow \infty ; \quad n \geqq 0 .
$$

Hence

$$
T_{1} \geqq 0, \quad T_{4}=O\left(\langle x\rangle^{-1}\right) \text { for } \quad x \rightarrow \infty .
$$

A computation shows that also

$$
T_{2} \geqq 0
$$

To deal with the term $T_{3}$ we notice that

$$
\nabla V \cdot \nabla\langle x\rangle=o(1) \text { for } \quad x \rightarrow \infty .
$$

Hence

$$
T_{3}=E \cdot \kappa \frac{x}{\langle x\rangle}+\varepsilon_{0}-\sum_{a: E^{a} \neq 0} \nabla V_{a}\left(x^{a}\right) \cdot E+o(1) ; \quad \varepsilon_{0}=|E|^{2} .
$$


For the first term we have

$$
\begin{aligned}
E \cdot \kappa \frac{x}{\langle x\rangle} & =\operatorname{Re}\left(\frac{\kappa}{\langle x\rangle}\left\{p^{2}+V-H\right\}\right) \\
& =p \cdot \frac{\kappa}{\langle x\rangle} p+\kappa\left(\frac{V}{\langle x\rangle}-\left(\Delta \frac{1}{2\langle x\rangle}\right)\right)-\operatorname{Re}\left(\frac{\kappa}{\langle x\rangle} H\right) \\
& \geqq-\operatorname{Re}\left(\frac{\kappa}{\langle x\rangle} H\right)+o(1) .
\end{aligned}
$$

Putting this together we obtain

$$
T_{2}+T_{3} \geqq \varepsilon_{0}-\sum_{a: E^{a} \neq 0} \nabla V_{a}\left(x^{a}\right) \cdot E-\operatorname{Re}\left(\frac{\kappa}{\langle x\rangle} H\right)+o(1) .
$$

Finally, by (4.1), (4.3) and (4.4),

$$
\begin{gathered}
i\left[H, e^{\theta} A_{f} e^{\theta}\right]=e^{\theta}\{\cdots\} e^{\theta} \\
\{\cdots\} \geqq T_{1}+\varepsilon_{0}-\sum_{b: E^{b} \neq 0} \nabla V_{b}\left(x^{b}\right) \cdot E-\operatorname{Re}\left(\frac{\kappa}{\langle x\rangle} H\right)+o(1) .
\end{gathered}
$$

In the following applications we consider some further specified functions $\theta$ depending on parameters. The estimate (4.5) will be uniform with respect to these parameters. As above we suppress the dependence of $\kappa$ in the notation for $f=f_{\kappa}$ and $\theta=\theta_{\kappa}\left(f_{k}\right)$.

Lemma 4.1. Let $\lambda \in \mathbf{R}, s>0$ and

$$
\theta(t)=s \log \left(t\left(1+\frac{t}{\mu}\right)^{-1}\right) ; \quad \mu>1 .
$$

Then for all sufficiently large $\kappa$ there exist a constant $C>0$ and a compact operator $K$ such that

$$
\begin{aligned}
\forall \mu & >1, \phi \in C_{0}^{\infty}(X):\left\langle\phi, i\left[(H-\lambda), e^{\theta} A_{f} e^{\theta}\right] \phi\right\rangle+C\left\|e^{\theta}(H-\lambda) \phi\right\|^{2} \\
& \geqq \frac{1}{C}\left\|e^{\theta} \phi\right\|^{2}-\left\|K e^{\theta} \phi\right\|^{2} .
\end{aligned}
$$

Lemma 4.2. Let $\lambda \in \mathbf{R}, \alpha_{0} \geqq 0$ and

$$
\theta(t)=\mu \log \left(1+\frac{\gamma t}{\kappa \mu}\right)+\frac{\alpha}{\kappa} t ; \quad \mu>1, \quad \gamma>0, \quad \alpha \geqq 0 .
$$

Then for a (small) $\delta>0$ and all sufficiently large $\kappa$ there exist a constant $C>0$ and a compact operator $K$ such that

$$
\begin{aligned}
& \forall \mu>1, \gamma+\left|\alpha-\alpha_{0}\right| \leqq \delta, \phi \in C_{0}^{\infty}(X): \\
& \quad\left\langle\phi, i\left[(H-\lambda), e^{\theta} A_{f} e^{\theta}\right] \phi\right\rangle+C\left\|e^{\theta}(H-\lambda) \phi\right\|^{2} \geqq \frac{1}{C}\left\|e^{\theta} \phi\right\|^{2}-\left\|K e^{\theta} \phi\right\|^{2} .
\end{aligned}
$$


Proof of Lemmas 4.1 and 4.2. We compute

$$
\theta^{\prime}(t) \leqq \frac{s}{t}, \quad\left|\theta^{\prime \prime}(t)\right| \leqq 2 \frac{s}{t^{2}}, \quad\left|\theta^{\prime \prime \prime}(t)\right| \leqq \frac{8 s}{t^{3}}
$$

in case of Lemma 4.1, while

$$
\left|\theta^{\prime}(t)-\frac{\alpha}{\kappa}\right| \leqq \frac{\gamma}{\kappa}, \quad\left|\theta^{\prime \prime}(t)\right| \leqq \frac{\gamma}{\kappa t}, \quad\left|\theta^{\prime \prime \prime}(t)\right| \leqq \frac{2 \gamma}{\kappa t^{2}}
$$

in case of Lemma 4.2 .

Thus by inspection we obtain for $h=T_{4}$ given by (4.1) that

$$
|h(x)| \leqq C\langle x\rangle^{-3} ; \quad C=C_{\kappa} \text { independent of } \mu,
$$

under the condition (4.6), while

$$
\begin{gathered}
|h(x)| \leqq C\langle x\rangle^{-1} ; \\
C=C_{\kappa} \text { independent of } \mu, \gamma, \alpha,
\end{gathered}
$$

under the conditions (4.7) and $\gamma+\left|\alpha-\alpha_{0}\right| \leqq 1$.

By (4.8) and (4.9) the fifth term on the right-hand side of (4.5) is $o(1)$ uniformly with respect to the parameters. Similarly all statements below will be uniform with respect to the parameter $\mu$ in case of Lemma 4.1 and $\mu, \gamma, \alpha$ in case of Lemma 4.2 (constrained as in the lemmas), and in addition with respect to $\phi \in C_{0}^{\infty}(X)$. For notational convenience we put $\alpha_{0}=0$ in case of Lemma 4.1 , introduce $\bar{\lambda}=$ $\lambda+\left(\alpha_{0}\right)^{2}$ and abbreviate the expectation $\left\langle e^{0} \phi, T e^{0} \phi\right\rangle=\langle T\rangle$ for any linear operator $T$.

As for the fourth term on the right-hand side of (4.5) we obtain from (4.6) and (4.7) that

$$
-\operatorname{Re}\left(\frac{\kappa}{\langle x\rangle} H\right)=-\operatorname{Re}\left(\frac{\kappa}{\langle x\rangle}\left\{H-\lambda-\left(\theta^{\prime 2}-\theta^{\prime \prime}\right)|\nabla f|^{2}\right\}\right)+O\left(\langle x\rangle^{-1}\right) .
$$

Hence by (4.2), the Cauchy Schwarz inequality and the same bounds again, it follows that for any $\phi \in C_{0}^{\infty}(X)$,

$$
\begin{aligned}
-\left\langle\operatorname{Re}\left(\frac{\kappa}{\langle x\rangle} H\right)\right\rangle & =-\operatorname{Re}\left\langle e^{\theta} \phi, \frac{\kappa}{\langle x\rangle} e^{\theta}(H-\lambda) \phi\right\rangle+\operatorname{Re}\left\langle\frac{2 \kappa i}{\langle x\rangle} \theta^{\prime} A_{f}\right\rangle+\left\langle O\left(\langle x\rangle^{-1}\right)\right\rangle \\
& \geqq-\kappa^{2}\left\|e^{\theta}(H-\lambda) \phi\right\|^{2}+\left\langle\kappa i\left[\frac{\theta^{\prime}}{\langle x\rangle}, A_{f}\right]\right\rangle+\left\langle O\left(\langle x\rangle^{-1}\right)\right\rangle \\
& \geqq-\kappa^{2}\left\|e^{\theta}(H-\lambda) \phi\right\|^{2}+\left\langle O\left(\langle x\rangle^{-1}\right)\right\rangle .
\end{aligned}
$$

As for the third term on the right-hand side of (4.5) we obtain by choosing $R$ large enough that it is estimated by

$$
\begin{aligned}
-\sum_{b: E^{b} \neq 0} h_{b}(x) & \geqq-\sum_{b: E^{b} \neq 0} F\left(\left|x^{b}\right|<R\right) h_{b}(x)-\frac{\varepsilon_{0}}{6} ; \\
h_{b}(x) & =\nabla V_{b}\left(x^{b}\right) \cdot E .
\end{aligned}
$$


We look closer at the first term on the right-hand side of (4.11). The idea is to write

$$
\begin{aligned}
F\left(\left|x^{b}\right|<R\right) & =\eta_{\varepsilon}(H-\bar{\lambda}) F\left(\left|x^{b}\right|<R\right)+\mathscr{E}_{b} ; \\
\mathscr{E}_{b} & =(H-\bar{\lambda}) \zeta_{\varepsilon}(H-\bar{\lambda}) F\left(\left|x^{b}\right|<R\right), \\
\zeta_{\varepsilon}(t) & =t^{-1}\left(1-\eta_{\varepsilon}(t)\right),
\end{aligned}
$$

and choose $\varepsilon$ small to treat the first term by Lemma 3.6 and its proof. The appearance of functions $h_{b}$ as factors is a minor complication since these are bounded. By mimicking the second step of the proof of Lemma 3.6 (cf. (3.2), (3.4), (3.11), (3.12) and Lemma 3.5 (2)) we can write

$$
\eta_{\varepsilon}(H-\bar{\lambda}) F\left(\left|x^{b}\right|<R\right)=\sum_{b \subset a} \eta_{\varepsilon}\left(H_{a}-\bar{\lambda}\right) F\left(\left|x^{b}\right|<R\right) j_{a}+K ; \quad K \text { compact } .
$$

The first term goes to zero as $\varepsilon \rightarrow 0$ since (3.13) holds as guaranteed by Lemma 3.6 and our induction hypothesis. In conclusion for $\varepsilon$ small enough

$$
-\operatorname{Re} \sum_{b: E^{b} \neq 0} \eta_{\varepsilon}(H-\bar{\lambda}) F\left(\left|x^{b}\right|<R\right) h_{b} \geqq-\frac{\varepsilon_{0}}{6} I-K ; \quad K \text { compact } .
$$

On the other hand for fixed $\varepsilon$ we can estimate

$$
\begin{aligned}
\left|\left\langle\mathscr{E}_{b} h_{b}\right\rangle\right| \leqq & \left\|(H-\bar{\lambda}) e^{\theta} \phi\right\| C\left\|e^{\theta} \phi\right\| \\
\leq & C^{\prime}\left\|(H-\bar{\lambda}) e^{\theta} \phi\right\|^{2}+\varepsilon^{\prime}\left\|e^{\theta} \phi\right\|^{2} \\
\leqq & 2 C^{\prime}\left\|\left(H-\lambda-\left(\theta^{\prime 2}-\theta^{\prime \prime}\right)|\nabla f|^{2}+2 i \theta^{\prime} A_{f}\right) e^{\theta} \phi\right\|^{2} \\
& +2 C^{\prime}\left\|\left(\left\{\left(\alpha_{0}\right)^{2}-\left(\theta^{\prime 2}-\theta^{\prime \prime}\right)|\nabla f|^{2}\right\}+2 i \theta^{\prime} A_{f}\right) e^{\theta} \phi\right\|^{2}+\varepsilon^{\prime}\left\|e^{\theta} \phi\right\|^{2} \\
& \varepsilon^{\prime}>0, \quad C^{\prime}=C_{\varepsilon, \varepsilon^{\prime}}^{\prime} .
\end{aligned}
$$

As for the first term we use (4.2). To estimate the second term we notice the bound

$$
\theta^{\prime} \leqq C \kappa^{-1}
$$

Moreover

$$
\left(\alpha_{0}\right)^{2}-\left(\theta^{\prime 2}-\theta^{\prime \prime}\right)|\nabla f|^{2}=O\left(\langle x\rangle^{-1}\right)
$$

under the condition (4.6), while

$$
\left.\left|\left(\alpha_{0}\right)^{2}-\left(\theta^{\prime 2}-\theta^{\prime \prime}\right)\right| \nabla f\right|^{2} \mid \leqq C\left(\langle x\rangle^{-1}+\kappa^{-1}+\delta\right)
$$

under the condition (4.7). In both cases $C$ is independent of $\kappa$ and the parameters (constrained to $\gamma+\left|\alpha-\alpha_{0}\right| \leqq \delta$ in case of Lemma 4.2). We apply the above estimates for $\kappa$ large, and in case of Lemma 4.2 for $\delta$ small. (Notice the bound $\left\|2 i \theta^{\prime} A_{f} e^{\theta} \phi\right\|^{2} \leqq \sup \theta^{\prime}\left\langle T_{1}\right\rangle$.) As a result

$$
\begin{aligned}
-\left\langle\operatorname{Re} \sum_{b: E^{b} \neq 0} \mathscr{E}_{b} h_{b}\right\rangle \geqq & -\frac{\varepsilon_{0}}{6}\left\|e^{\theta} \phi\right\|^{2}-C\left\|e^{\theta}(H-\lambda) \phi\right\|^{2} \\
& -\left\langle\frac{T_{1}}{2}\right\rangle+\left\langle O\left(\langle x\rangle^{-2}\right)\right\rangle .
\end{aligned}
$$


By combining (4.5), (4.10), (4.11), (4.13) and (4.14) we finally obtain that for a constant $C>0$ and compact $K$ (both independent of $\mu$, and in case of Lemma 4.2 also of $\gamma$ and $\alpha$ )

$$
\begin{aligned}
& \left\langle\phi, i\left[(H-\lambda), e^{\theta} A_{f} e^{\theta}\right] \phi\right\rangle+C\left\|e^{\theta}(H-\lambda) \phi\right\|^{2} \\
& \quad \geqq \frac{\varepsilon_{0}}{2}\left\|e^{\theta} \phi\right\|^{2}+\left\langle\frac{T_{1}}{2}\right\rangle-\left\|K e^{\theta} \phi\right\|^{2}+\langle\tilde{h}\rangle ; \quad \tilde{h}=o(1) .
\end{aligned}
$$

It remains to treat the last term on the right-hand side of (4.15). For that we pick $C^{\prime}, R>1$ independent of the parameters such that

$$
\tilde{h} \geqq-C^{\prime} F(|x|<R)-\frac{\varepsilon_{0}}{6} ;
$$

cf. (4.8) and (4.9). Since for any $\sigma>0$ the operator $\eta_{\sigma}(H) F(|x|<R)$ is compact we can estimate

$$
-C^{\prime} F(|x|<R) \geqq-K-C^{\prime} \operatorname{Re}\left(H \zeta_{\sigma}(H) F(|x|<R)\right),
$$

where $K$ is compact and $\zeta_{\sigma}$ is given by (4.12).

By an argument now familiar there exists a constant $\tilde{C}>0$ such that

$$
\tilde{C}^{-1}\left\|H e^{\theta} \phi\right\|^{2} \leqq\left\|e^{\theta} \phi\right\|^{2}+\left\|e^{\theta}(H-\lambda) \phi\right\|^{2}+\left\langle T_{1}\right\rangle .
$$

By choosing $\sigma$ large enough we obtain from the bound $\left\|\zeta_{\sigma}(H)\right\| \leqq \sigma^{-1}$ and (4.18) that

$$
-C^{\prime} \operatorname{Re}\left\langle H \zeta_{\sigma}(H) F(|x|<R)\right\rangle \geqq-\frac{\varepsilon_{0}}{6}\left\|e^{0} \phi\right\|^{2}-\left\langle\frac{T_{1}}{2}\right\rangle-\left\|e^{0}(H-\lambda) \phi\right\|^{2} .
$$

Putting together the statements (4.15),(4.16),(4.17) and (4.19) it follows that

$$
\left\langle\phi, i\left[(H-\lambda), e^{\theta} A_{f} e^{\theta}\right] \phi\right\rangle+C\left\|e^{\theta}(H-\lambda) \phi\right\|^{2} \geqq \frac{\varepsilon_{0}}{6}\left\|e^{\theta} \phi\right\|^{2}-\left\|K e^{\theta} \phi\right\|^{2} .
$$

Hence we have proved the lemmas.

The previous estimates lead to

Lemma 4.3. Suppose $(H-\lambda) \psi=0 ; \lambda \in \mathbf{R}, \psi \in \mathscr{D}(H)$. Then

$$
\forall \alpha \geqq 0: e^{\alpha\langle x\rangle} \psi(x) \in L^{2}(X) .
$$

Proof. First we prove

$$
\forall s \geqq 0:\langle x\rangle^{s} \psi(x) \in L^{2}(X) .
$$

For that we use Lemmas 3.1 and 4.1 to prove by an approximation argument that

$$
C\left\|K e^{0} \psi\right\|^{2} \geqq\left\|e^{0} \psi\right\|^{2},
$$

where $C, K$ and $\theta$ are as in Lemma 4.1. If (4.20) does not hold we obtain a contradiction by letting $\mu \rightarrow \infty$ in (4.21).

Now let

$$
\alpha_{0}=\sup \left\{\alpha \geqq 0 \mid e^{\alpha\langle x\rangle} \psi(x) \in L^{2}(X)\right\} .
$$

We need to show that $\alpha_{0}=\infty$. Suppose this statement is false. 
If $\alpha_{0}=0$ we come to a contradiction by using (4.20), Lemma 3.2, and Lemma 4.2 with $\alpha=\alpha_{0}$. It is obtained by letting $\mu \rightarrow \infty$ in an estimate similar to (4.21).

If $\alpha_{0}>0$ the contradiction arises by the following use of Lemma 4.2. Let $\delta>0$ be given in accordance with the lemma. Then we choose any $\alpha<\alpha_{0}$ such that $\alpha_{0}<\alpha+\frac{\delta}{2}$ and put $\gamma=\frac{\delta}{2}$. It remains to specify $\kappa$. We choose it so large that in addition to the estimate of the lemma the conditions

$$
\alpha\left(1+\frac{|E|}{\kappa}\right)<\alpha_{0} \text { and } \alpha_{0}<\left(\alpha+\frac{\delta}{2}\right)\left(1-\frac{|E|}{\kappa}\right)
$$

hold. Now we proceed as above, that is by first using Lemma 3.2 to replace $\phi$ in the estimate by $\psi$ and then letting $\mu \rightarrow \infty$.

Proof of Theorem 2.2. By using Lemmas 3.2 and 4.3 the statement follows readily from the following formula valid for any $\phi \in C_{0}^{\infty}(X)$ :

$$
\begin{aligned}
& \left\|e^{\alpha E \cdot x}\{(H-\lambda)-V\} \phi\right\|^{2} \\
& =\left\|\left\{p^{2}-E \cdot x-\left(\lambda+\alpha^{2}|E|^{2}\right)\right\} e^{\alpha E \cdot x} \phi\right\|^{2} \\
& +4 \alpha^{2}\left\|E \cdot p e^{\alpha E \cdot x} \phi\right\|^{2}+2 \alpha|E|^{2}\left\|e^{\alpha E \cdot x} \phi\right\|^{2} ; \quad \lambda \in \mathbf{R}, \quad \alpha \geqq 0 .
\end{aligned}
$$

Because of Lemma 4.3 we can apply it to $\phi=\psi$ if $(H-\lambda) \psi=0$. This gives the condition

$$
\left\|e^{\alpha E \cdot x} V \psi\right\|^{2} \geqq 2 \alpha|E|^{2}\left\|e^{\alpha E \cdot x} \psi\right\|^{2},
$$

which clearly holds for all $\alpha \geqq 0$ only if $\psi=0$.

\section{Section 5. Proof of Theorem 2.3}

We shall outline a proof of Theorem 2.3. As for the first statement (1) of the theorem we use the Mourre method [M] (see also [PSS]) with the conjugate operator $A=E \cdot p$. Indeed the Mourre estimate holds for this example in fact uniformly with respect to translations of the energy: Let $\rho<|E|^{2}$. Then for $\varepsilon>0$ small enough

$$
\eta_{\varepsilon}(H-\lambda) i[H, A] \eta_{\varepsilon}(H-\lambda) \geqq \rho \eta_{\varepsilon}(H-\lambda)^{2} ; \quad \lambda \in \mathbf{R} .
$$

This statement follows easily from the computations in the beginning of Sect. 4 in conjunction with Theorem 2.2 and Lemma 3.6. In order to apply the abstract theory of $[\mathrm{M}]$ one needs in addition to the positivity statement (5.1) a property of the double commutator $[[H, A], A]$. In the present context this commutator is a bounded function by Condition 2.1 (2) and hence in accordance with the assumptions made in [M]. It is now a matter of mimicking [M] and [PSS] to obtain (1) with the weight $\langle p\rangle^{-\delta}$ replaced by $\langle A\rangle^{-\delta}$. We apply (5.1) with $\lambda=\operatorname{Re} z$, where $z$ is the resolvent parameter. Since $\langle A\rangle^{\delta}\langle p\rangle^{-\delta}$ is bounded we can then obtain the resolvent estimate with the weight $\langle p\rangle^{-\delta}$ as stated. The statement about existence of weighted boundary values with this weight follows by the same methods.

To obtain the part of Theorem 2.3 that involves the weight $\langle x\rangle^{-\delta^{\prime}}$ one can use Lemma 3.1 to replace $\langle x\rangle^{-\delta^{\prime}}$ by $\langle p\rangle^{-2 \delta^{\prime}}$ and thus reduce to the previous part. We omit the details referring the reader to [Sk] where in particular a detailed proof of 
the low energy bound of (2) can be found. As for the high energy bound it suffices to show that for any $\frac{1}{4}<\delta^{\prime} \leqq \frac{1}{2}$,

$$
\left\|\langle p\rangle^{2 \delta^{\prime}}(H-\lambda-i)^{-1}\langle x\rangle^{-\delta^{\prime}}\right\| \leqq C\langle\lambda\rangle^{\delta^{\prime}} ; \quad \lambda \geqq 0 .
$$

This result follows by interpolating the result for $\delta^{\prime}=\frac{1}{2}$ which in turn follows readily by a commutation and by the method used in the proof of Lemma 3.1.

\section{Section 6. Coulomb Singularities}

In this section we extend our results to cover particles interacting through Coulomb forces. We will make use of the following conditions on the potential all of which are satisfied for the usual models for atoms and molecules.

\section{Conditions 6.1.}

(1) For all $b \in \mathscr{A}$ the real measurable potential $V_{b}$ obeys

$$
\left|V_{b}\left(x^{b}\right)\right| \leqq C_{b} \sum_{j=1}^{k}\left|x^{b}-r_{\jmath}\right|^{-1}+o(1),
$$

with $r_{1}, \ldots, r_{k}$ all in $X^{b}$, and has distributional gradient satisfying

$$
\left|\nabla V_{b}\left(x^{b}\right)\right| \leqq C_{b} \sum_{j=1}^{k}\left|x^{b}-r_{j}\right|^{-2}+o(1),
$$

where $o(1)$ is a bounded function which tends to zero as $x^{b} \rightarrow \infty$.

(2) Outside a compact set in $X^{a}, V_{a}$ is $C^{1}$ with bounded second order derivatives.

(C) If $V_{a} \neq 0, \Pi^{a} \Pi^{b} \neq 0$, and $C_{b}$ in (1) above is non-zero, then $\operatorname{dim}$ (Range $\left.\Pi^{a} \Pi^{b}\right) \geqq 3$.

We believe 6.1 (C) in unnecessary for $a \neq b$. Although we will not show it, it is unnecessary if the singularity in the potential and its derivative are slightly weaker in an $L^{p}$ sense. This can be proved with the techniques introduced below in conjunction with Appendix B (or alternatively in conjunction with the ideas of $[\mathrm{ABG}, \mathrm{T} 4])$.

Our main results of this section are

Theorem 6.2. If Conditions 6.1 (1) and (C) are satisfied and $E \neq 0$, then $\sigma_{p p}(H)$ is empty.

Theorem 6.3. If Conditions 6.1 are satisfied and $E \neq 0$ then $\sigma_{s c}(H)=\emptyset$. In fact for $\delta>\frac{1}{2}$ and $\delta^{\prime}>\frac{1}{4}$ the limits $\left(\right.$ in $\mathscr{B}\left(L^{2}(X)\right)$ )

$$
\lim _{\operatorname{Im} z \downarrow(\uparrow) 0}\langle p\rangle^{-\delta}(H-z)^{-1}\langle p\rangle^{-\delta}, \quad \lim _{\operatorname{Im} z \downarrow(\uparrow) 0}\langle x\rangle^{-\delta^{\prime}}(H-z)^{-1}\langle x\rangle^{-\delta^{\prime}}
$$

are attained uniformly for $\operatorname{Re} z$ in compact sets.

For the proofs we need some preliminaries.

In the presence of an electric field, singularities present a much more challenging and interesting problem than with the usual Schrödinger operator. This 
is because one is forced to prove bounds uniform in the spectral parameter. Thus, if $\Pi$ is an orthogonal projection with $(1-\Pi) E \neq 0$, one readily observes that

$$
\left\|V(\Pi x)\left(H_{0}-\mu-i \gamma\right)^{-1}\right\|=\sup _{\lambda \in \mathbf{R}}\left\|V(y)\left(-\Delta_{y}-\Pi E \cdot y-\lambda-i \gamma\right)^{-1}\right\|
$$

As the following proposition makes clear, the Coulomb potential is on the borderline for $H_{0}$-boundedness.

Proposition 6.4. Suppose $\Pi$ is an orthogonal projection onto a subspace of $X$ with dimension $\geqq 3$. Then

(1)

$$
|||\Pi x|^{-1}\left(H_{0}-\lambda-i \gamma\right)^{-1} \| \leqq C_{1}|\gamma|^{-\frac{1}{2}},
$$

where $C_{1}$ is independent of $\gamma, \lambda$, and $E$.

(2) If $V$ is a measurable function on $\Pi X$ with the property that

$$
\lim _{\Pi x \rightarrow 0}|V(\Pi x) \Pi x|=\infty
$$

and if $(1-\Pi) E \neq 0$ then $V(\Pi x)$ is not $H_{0}$-bounded.

Remarks. (1) If we define $|\Pi x|^{-1} \delta\left(H_{0}-\lambda\right)|\Pi x|^{-1}$ as a form on $C_{0}^{\infty}(X) \times C_{0}^{\infty}(X)$ by the formula

$$
\begin{aligned}
\left\langle\varphi,|\Pi x|^{-1} \delta\left(H_{0}-\lambda\right)|\Pi x|^{-1} \psi\right\rangle= & \lim _{\varepsilon \downarrow 0}(2 \pi i)^{-1}\left\langle\varphi,\left[\left(H_{0}-\lambda-i \varepsilon\right)^{-1}\right.\right. \\
& \left.\left.-\left(H_{0}-\lambda+i \varepsilon\right)^{-1}\right] \psi\right\rangle
\end{aligned}
$$

then (1) of Proposition 6.4 is equivalent to the estimate

$$
\left\||\Pi x|^{-1} \delta\left(H_{0}-\lambda\right)|\Pi x|^{-1}\right\| \leqq \frac{C_{1}^{2}}{\pi} .
$$

The existence of the limit above can be inferred from the proof of (1).

(2) Note that by scaling, the uniformity in $E$ of the bound in (1) of Proposition 6.4 follows from the uniformity in $\lambda$.

Proposition 6.4 is proved in Appendix A, but see the remark after the end of the proof of Lemma 6.5 for a simple proof of a weaker version.

We will make use of the following:

\section{Lemma 6.5.}

(1) Suppose $Y$ is a subspace contained in $X$ and $f \in L^{p}(Y)$ with $p \geqq 2$ and $p>d=\operatorname{dim} Y$. Suppose $\frac{d}{2 p}<\alpha \leqq 1$. Let $\Pi$ be the orthogonal projection onto $Y$. Then

$$
\left\|f(\Pi x)\left|H_{0}-\lambda-i \gamma\right|^{-\alpha}\right\| \leqq C(\alpha, d, p)|\gamma|^{-(\alpha-d / 2 p)}\|f\|_{L^{p}(Y)} .
$$

(2) Fix $a, b \in \mathscr{A}$ such that $\Pi^{a} \Pi^{b} \neq 0$. Define

$$
V_{\bar{b}}=\sum_{\Pi^{c} \Pi^{b}=0} V_{c}, \quad H_{\tilde{b}}=H_{0}+V_{\tilde{b}}, \quad \text { and } \quad Y_{b a}=X^{b} \ominus\left(X^{b} \cap X_{a}\right) .
$$


Suppose $p \geqq 2$ and $2 p>d=\operatorname{dim} Y_{b a}$. Let $f$ and $g$ be measurable functions on $X^{a}$ and $X^{b}$, respectively. Then

$$
\left\|f\left(\Pi^{a} x\right)\left(H_{\tilde{b}}-\lambda-i \gamma\right)^{-2} g\left(\Pi^{b} x\right)\right\| \leqq C(d, p, a, b)|\gamma|^{-(2-d / p)} A_{f} B_{g},
$$

where

$$
\begin{aligned}
& A_{f}=\sup _{w \in X^{a}}\left(\int_{\Pi^{a} X^{b}}|f(u+w)|^{p} d u\right)^{\frac{1}{p}}, \\
& B_{g}=\sup _{z \in X^{b} \cap X_{a}}\left(\int_{Y_{b a}}|g(y+z)|^{p} d y\right)^{\frac{1}{p}} .
\end{aligned}
$$

Remarks.

(1) Note that part (1) provides a large class of $H_{0}$-bounded potentials, but that the Coulomb singularity just misses being included. Nevertheless the bound will be useful. For a somewhat similar result (proved by the same technique) we refer to [K, Lemma 3.1].

(2) Part (2) is a technical result which is crucial to our treatment of the Coulomb singularity. Note that $A_{f}$ is finite if $f$ is bounded with compact support.

Proof. By (3.1),

$$
\left\|f(\Pi x) e^{-t t\left(H_{0}-i-l_{i}\right)} f(\Pi x)\right\|=\left\|f e^{i t \Delta_{Y}} f\right\| e^{-t \gamma},
$$

where the norm on the right is in $L^{2}(Y)$. A standard estimate [RS, p. 154],

$$
\left\|f e^{i t \Delta_{Y}} f\right\| \leqq\|f\|_{L^{p}(Y)}^{2}(4 \pi t)^{-d / p}, \quad t>0,
$$

then gives (for $\gamma>0$ )

$$
\left\|f\left(H_{0}-\lambda-i \gamma\right)^{-1} f\right\| \leqq 2|\gamma|^{-(1-d / p)}(1-d / p)^{-1}\|f\|_{L^{p}(Y)}^{2}
$$

after integration over $t$. It follows that

$$
\begin{aligned}
\left\|f\left(\left(H_{0}-\lambda\right)^{2}+\gamma^{2}\right)^{-\frac{1}{2}}\right\|^{2} & =\left\|f\left(\left(H_{0}-\lambda\right)^{2}+\gamma^{2}\right)^{-1} f\right\| \\
& =(2|\gamma|)^{-1}\left\|f\left[\left(H_{0}-\lambda-i \gamma\right)^{-1}-\left(H_{0}-\lambda+i \gamma\right)^{-1}\right] f\right\| \\
& \leqq 2|\gamma|^{-2(1-d / 2 p)}(1-d / p)^{-1}\|f\|_{L^{p}(Y)}^{2} .
\end{aligned}
$$

This proves (1) for $\alpha=1$. Part (1) (for $\frac{d}{2 p}<\alpha<1$ ) then follows by using the formula

$$
\left(\left(H_{0}-\lambda\right)^{2}+\gamma^{2}\right)^{-\alpha / 2}=C_{\alpha} \int_{0}^{\infty}\left(\left(H_{0}-\lambda\right)^{2}+\gamma^{2}+\mu^{2}\right)^{-1 / 2} \mu^{-\alpha} d \mu
$$

and keeping track of the constants.

The proof of (2) is similar except that we use $[\mathrm{RS}, \mathrm{IO}]$

$$
\begin{aligned}
\left\|f\left(\Pi^{a} x\right) e^{-i t\left(H_{b^{-}}-\lambda-i_{\gamma}\right)} g\left(\Pi^{b} x\right)\right\| & =\left\|f\left(\Pi^{a} x\right) e^{i t \Delta_{b a}} g\left(\Pi^{b} x\right)\right\| e^{-t_{\gamma}} \\
& \leqq \tilde{A}_{f} B_{g} e^{-t \gamma^{\prime}}(4 \pi t)^{-d / p}, \quad t>0 .
\end{aligned}
$$


Here

$$
\tilde{A}_{f}=\sup _{x_{b} \in X_{b}}\left(\int_{Y_{b a}}\left|f\left(\Pi^{a} y+\Pi^{a} x_{b}\right)\right|^{p} d y\right)^{\frac{1}{p}}=C^{-\frac{1}{p}} A_{f},
$$

where $C$ is the relevant Jacobian.

We now multiply by $t$ and integrate to obtain the result for $\gamma>0$.

Remark. The methods used to prove Lemma 6.5 yield a simple proof of a weaker version of the first inequality of Proposition 6.4, namely that for fixed $E$ and any $\varepsilon>0$

$$
\left\||\Pi x|^{-1}\left(H_{0}-\lambda-i \gamma\right)^{-1}\right\| \leqq C_{\varepsilon}|\gamma|^{-\frac{1}{2}}\left(1+|\gamma|^{-\varepsilon}\right) .
$$

In proving this there is no loss of generality in assuming $\Pi=I$. Then Lemma 6.5(1) with $f=F(|x| \geqq 1)|x|^{-1}$ and $p=d /(1-2 \varepsilon)$ (with $\varepsilon>0$ and small) yields

$$
\left\|F(|x| \geqq 1)|x|^{-1}\left(H_{0}-\lambda-i \gamma\right)^{-1}\right\| \leqq C_{\varepsilon}|\gamma|^{-\frac{1}{2}-\varepsilon} .
$$

Now let $f=F(|x|<1)|x|^{-1}, \eta(\lambda)=\left(\lambda^{2}+\gamma^{2}\right)^{-1}$. We obtain

$$
f \eta\left(H_{0}-\lambda\right) f=\frac{1}{\sqrt{2 \pi}} \int \hat{\eta}(t) e^{-i|E|^{2} t^{3} / 12} e^{i t E \cdot x / 2} f e^{-i t\left(p^{2}-\lambda\right)} f e^{i t E \cdot x / 2} d t .
$$

Using the estimates $\left|e^{-i|E|^{2} t^{3} / 12}-1\right| \leqq C|E|^{2 \delta / 3}|t|^{\delta}$ and $\left|e^{i t E \cdot x / 2}-1\right| \leqq C|E|^{\delta}$ $|x|^{\delta}|t|^{\delta}$ for $\delta \in(0,1]$, we find

$$
\left\|f \eta\left(H_{0}-\lambda\right) f-f \eta\left(p^{2}-\lambda\right) f\right\| \leqq C\|f\|_{L^{p}(X)}^{2} \int|t|^{\delta}|t|^{-d / p}|\hat{\eta}(t)| d t .
$$

We take $\delta=3 \varepsilon, p=d /(1+\varepsilon)$ (with $\varepsilon>0$ and small) and obtain

$$
\left\|f \eta\left(H_{0}-\lambda\right) f-f \eta\left(p^{2}-\lambda\right) f\right\| \leqq C_{\varepsilon}|\gamma|^{-1-2 \varepsilon} .
$$

We have thus reduced the problem to the case where $E=0$. We have

$$
\begin{aligned}
|\gamma|\left\|f \eta\left(p^{2}-\lambda\right) f\right\| & \leqq \frac{1}{2}\left\||x|^{-1}\left\{\left(p^{2}-\lambda-i \gamma\right)^{-1}-\left(p^{2}-\lambda+i \gamma\right)^{-1}\right\}|x|^{-1}\right\| \\
& \leqq\left\||x|^{-1}\left(p^{2}-\lambda-i \gamma\right)^{-1}|x|^{-1}\right\| .
\end{aligned}
$$

We learned an especially simple way of estimating the above norm from Agmon [A]: First we can assume without loss that $d=3$. (If $d>3$ write $x=$ $(y, z)$ with $y \in \mathbf{R}^{3}$ and estimate $|x|^{-1} \leqq|y|^{-1}$.) In three dimensions the operator $\left(p^{2}-\lambda-i \gamma\right)^{-1}$ has an integral kernel $(4 \pi|x-y|)^{-1} \exp (i \sqrt{\lambda+i \gamma}|x-y|)$, which is pointwise bounded by the integral kernel of $(-\Delta)^{-1}$. Thus, by Hardy's inequality $\left(|p|^{2} \geqq \frac{1}{4|x|^{2}}\right)$ we have

$$
\sup _{\lambda \in \mathbf{R}}|\gamma|\left\|f \eta\left(p^{2}-\lambda\right) f\right\| \leqq 4,
$$

and we thus obtain

$$
\left\|f \eta\left(H_{0}-\lambda\right) f\right\| \leqq C\left(|\gamma|^{-1-2 \varepsilon}+|\gamma|^{-1}\right),
$$

which gives the result.

Let $\mathscr{A}^{\prime}=\mathscr{A} \backslash\left\{a_{\min }\right\}$. 
Lemma 6.6. If Condition 6.1 (1) is satisfied, there exists for each a $\in \mathscr{A}^{\prime}$ a sequence a real-valued functions $V_{a}^{(m)} \in C_{0}^{\infty}\left(X^{a}\right)$ such that if

$$
H_{c}^{(m)}=H_{0}+\sum_{b \subset c} V_{b}^{(m)}
$$

then

$$
\lim _{m \rightarrow \infty} \sup _{\lambda \in \mathbf{R}}\left\|\left(H_{c}-\lambda-i \gamma\right)^{-1}-\left(H_{c}^{(m)}-\lambda-i \gamma\right)^{-1}\right\|=0
$$

for each $\gamma \in \mathbf{R} \backslash\{0\}$ and each $c \in \mathscr{A}^{\prime}$.

Proof. We prove the result for $c=a_{\max }$. Introduce the matrix

$$
M_{a b}(z)=W_{a}\left(z-H_{0}\right)^{-1} \underline{W}_{b}, \quad \operatorname{Im} z \neq 0,
$$

where $W_{a}=\left|V_{a}\right|^{\frac{1}{2}}, \underline{W}_{a}=\left|V_{a}\right|^{\frac{1}{2}} \operatorname{sgn} V_{a}$. It follows from Lemma 6.5 (1) (with $\alpha=\frac{1}{2}$ ) that on the Hilbert space

$$
\mathscr{H}=\bigoplus_{a \in \mathscr{C l}^{\prime}} L^{2}\left(\mathbf{R}^{n}\right)
$$

we have

$$
\|M(z)\| \leqq \frac{1}{2}
$$

if $|\operatorname{Im} z|$ is large enough. As in [H1, Lemma 3.4] (cf. also [RS, p. 152, IO]) we have for $|\operatorname{Im} z|$ large

$$
(z-H)^{-1}=\left(z-H_{0}\right)^{-1}+\sum_{a, b}\left(z-H_{0}\right)^{-1} \underline{W}_{a}(I-M(z))_{a b}^{-1} W_{b}\left(z-H_{0}\right)^{-1} .
$$

Again, using Lemma 6.5 (1) and with the help of (6.2) we can approximate $W_{a}$ and $\underline{W}_{a}$ by real functions in $C_{0}^{\infty}\left(X^{a}\right)$ to show (6.1) for $|\gamma|$ large. But then (6.1) follows for all $\gamma \neq 0$ using the resolvent equation.

Corollary 6.7. If Condition 6.1 (1) is satisfied, then Lemmas 3.5 and 3.6 are also true for the potentials being considered here.

Proof. Lemma 3.5 is valid for the Hamiltonians $H_{a}^{(m)}$. We use a simple approximation argument based on Lemma 6.6 to finish the proof. The proof of Lemma 3.6 uses only Lemma 3.5 .

Lemma 3.6 is not strong enough for us in the presence of the Coulomb singularity. We need the following.

Lemma 6.8. Suppose Conditions 6.1 (1) and (C) are satisfied and $b \in \mathscr{A}$ is given with $C_{b} \neq 0$. Let $g\left(x^{b}\right)=\left|x^{b}-r\right|^{-1}$ with $r \in X^{b}$. Then

$$
\lim _{\varepsilon, \varepsilon^{\prime} \backslash 0}\left\|\eta_{\varepsilon}(H-\lambda) g\left(x^{b}\right) F\left(\left|x^{b}-r\right|<\varepsilon^{\prime}\right)\right\|=0
$$

uniformly in $\lambda$.

Proof. Let $B=\eta_{\varepsilon}(H-\lambda) g\left(x^{b}\right) F\left(\left|x^{b}-r\right|<\varepsilon^{\prime}\right)$ and let $\tilde{b} \in \mathscr{A}$ be given as in Lemma 6.5 (2). Note that by inserting $1=\left(H_{\tilde{b}}-\lambda-i \gamma\right)^{2}\left(H_{\tilde{b}}-\lambda-i \gamma\right)^{-2}$ we have

$$
B=-\gamma^{2} \eta_{\varepsilon}(H-\lambda)\left(H_{\tilde{b}}-\lambda-i \gamma\right)^{-2} g\left(x^{b}\right) F\left(\left|x^{b}-r\right|<\varepsilon^{\prime}\right)+\mathscr{E}_{\gamma},
$$


where $\left\|\mathscr{E}_{\gamma}\right\| \rightarrow 0$ as $\gamma \rightarrow \infty$ uniformly in $\lambda, \varepsilon$, and $\varepsilon^{\prime}$ for $\varepsilon \leqq 1$. This follows from Proposition 6.4. Thus we need only show that for fixed $\gamma>0$ the operator

$$
B^{\prime}=\eta_{\varepsilon}(H-\lambda)\left(H_{\tilde{b}}-\lambda-i \gamma\right)^{-2} g\left(x^{b}\right) F\left(\left|x^{b}-r\right|<\varepsilon^{\prime}\right)
$$

tends to 0 in norm uniformly in $\lambda$ as $\varepsilon, \varepsilon^{\prime} \rightarrow 0$. If in the expression for $B^{\prime}, \eta_{\varepsilon}(H-\lambda)$ is replaced by $\eta_{\varepsilon}\left(H_{\tilde{b}}-\lambda\right)$, the result is true, because

$$
\begin{aligned}
& \sup _{\lambda \in \mathbf{R}}\left\|\eta_{\varepsilon}\left(H_{\tilde{b}}-\lambda\right) g\left(x^{b}\right)\right\|^{2} \leqq \sup _{\mu \in \mathbf{R}}\left\|g\left(x^{b}\right) \eta_{\varepsilon}^{2}\left(\left(p^{b}\right)^{2}-E^{b} \cdot x^{b}-\mu\right) g\left(x^{b}\right)\right\| \\
& \quad \leqq \sup _{\mu \in \mathbf{R}} \int\left\|g\left(x^{b}\right) \delta\left(\left(p^{b}\right)^{2}-E^{b} \cdot x^{b}-\mu-t\right) g\left(x^{b}\right)\right\| \eta_{\varepsilon}^{2}(t) d t \leqq C \varepsilon .
\end{aligned}
$$

The last inequality follows from Proposition 6.4 (1) by a simple translation argument. Thus it suffices to show that for fixed $\varepsilon>0$,

$$
\left\|\left[\eta_{\varepsilon}(H-\lambda)-\eta_{\varepsilon}\left(H_{\tilde{b}}-\lambda\right)\right]\left(H_{\tilde{b}}-\lambda-i \gamma\right)^{-2} g\left(x^{b}\right) F\left(\left|x^{b}-r\right|<\varepsilon^{\prime}\right)\right\| \rightarrow 0,
$$

uniformly in $\lambda$ as $\varepsilon^{\prime} \downarrow 0$. By Lemma 6.6 we can approximate $\eta_{\varepsilon}(H-\lambda)-\eta_{\varepsilon}\left(H_{\tilde{b}}-\right.$ $\lambda)$ by $\eta_{\varepsilon}\left(H^{(m)}-\lambda\right)-\eta_{\varepsilon}\left(H_{\tilde{b}}^{(m)}-\lambda\right)$, where the superscript $m$ indicates that each nonzero $V_{a}$ has been replaced by a function in $C_{0}^{\infty}\left(X^{a}\right)$. We now proceed as in the proof of Lemma 3.5, first approximating $\eta_{\varepsilon}\left(H^{(m)}-\lambda\right)-\eta_{\varepsilon}\left(H_{\tilde{b}}^{(m)}-\lambda\right)$ using the Stone Weierstrass theorem, and then expanding the resolvents which occur in a Neumann series. Because we are dealing with the difference above, each term will contain a potential $V_{a}^{(m)} \in C_{0}^{\infty}\left(X^{a}\right)$, where $\Pi^{a} \Pi^{b} \neq 0$. Arguing as in the proof of Lemma 3.5, we are reduced to estimating

$$
\left\|f\left(x^{a}\right)\left(H_{\tilde{b}}-\lambda-i \gamma\right)^{-2} g\left(x^{b}\right) F\left(\left|x^{b}-r\right|<\varepsilon^{\prime}\right)\right\|,
$$

where $f$ is bounded with compact support. To obtain this estimate we use Lemma 6.5 (2). We must estimate $B_{g F}$ with $\operatorname{dim} Y_{b a}=d \geqq 3$. But if $x^{b}=y+z$, with $y \in$ $Y_{b a}$ and $z \in X^{b} \cap X_{a}$ we have $g\left(x^{b}\right) \leqq\left|y-r^{\prime}\right|^{-1}$ with $r^{\prime}$ the projection of $r$ on $Y_{b a}$. Thus choosing $p \in\left(\frac{d}{2}, d\right)$ with $p \geqq 2$ it is obvious that as $\varepsilon^{\prime} \downarrow 0$,

$$
B_{g F} \leqq\left(\int_{Y_{b a}} F\left(\left|y-r^{\prime}\right|<\varepsilon^{\prime}\right)\left|y-r^{\prime}\right|^{-p} d y\right)^{\frac{1}{p}} \rightarrow 0 .
$$

We are now ready to give the

Proof of Theorem 6.2. We follow the proof of Theorem 2.2 making adjustments when necessary to deal with the singularities in $V$ and $\nabla V$. Our first task is to prove the estimates of Lemmas 4.1 and 4.2. For notational convenience we assume for all $b \in \mathscr{A}$ with $C_{b} \neq 0$ (see Condition 6.1 (1)) that $k=1$ and $r_{1}=0$. We first redo the calculations leading to $(4.5)$ with the result

$$
\begin{gathered}
i\left[H, e^{\theta} A_{f} e^{\theta}\right]=e^{\theta}\{\cdots\} e^{\theta} . \\
\{\cdots\} \geqq T_{1}+\varepsilon_{0}-\sum_{b: E^{b} \neq 0}\left(\nabla V_{b}\left(x^{b}\right) \cdot E\right) F\left(\left|x^{b}\right| \geqq \varepsilon_{1}\right) \\
-\operatorname{Re}\left(\frac{x}{\langle x\rangle} H\right)+o(1)-C \sum_{b \in \mathscr{A}}\left(\left|V_{b}\left(x^{b}\right)\right|+\left|\nabla V_{b}\left(x^{b}\right)\right|\right) F\left(\left|x^{b}\right|<\varepsilon_{1}\right) .
\end{gathered}
$$


Here $o(1)$ depends on $\varepsilon_{1}>0$. It denotes a bounded function which tends to zero as $x \rightarrow \infty$. In order to obtain (6.3) we have used the simple estimate

$$
\left|\frac{x \cdot \nabla V}{\langle x\rangle}\right| \leqq \sum_{b \in \mathscr{d}}\left|\nabla V_{b}\left(x^{b}\right)\right| F\left(\left|x^{b}\right|<\varepsilon_{1}\right)+o(1) \text {. }
$$

As in Sect. 4, let $\bar{\lambda}=\lambda+\alpha_{0}^{2}$ and write $\eta_{\varepsilon_{2}}=\eta_{\varepsilon_{2}}(H-\bar{\lambda})$. Let $g_{b}=\left|V_{b}\left(x^{b}\right)\right|+$ $\left|\nabla V_{b}\left(x^{b}\right)\right|, F_{b}=F\left(\left|x^{b}\right|<\varepsilon_{1}\right)$. We consider the last term of $(6.3)$ :

$$
\sum_{b \in . \mathscr{d}}\left\langle g_{b} F_{b}\right\rangle=\sum_{b \in \mathscr{O}}\left(\left\langle\eta_{\varepsilon_{2}} g_{b} F_{b} \eta_{\varepsilon_{2}}\right\rangle+\left\langle\left(1-\eta_{\varepsilon_{2}}\right) g_{b} F_{b} \eta_{\varepsilon_{2}}\right\rangle+\left\langle g_{b} F_{b}\left(1-\eta_{\varepsilon_{2}}\right)\right\rangle\right) .
$$

Lemmas 6.8 and 3.6 control the first term. We choose $\varepsilon_{1}$ and $\varepsilon_{2}$, so that

$$
\sum_{b \in . \mathscr{O}}\left|\left\langle\eta_{\varepsilon_{2}} g_{b} F_{b} \eta_{\varepsilon_{2}}\right\rangle\right| \leqq \delta^{\prime}\left\|e^{\theta} \phi\right\|^{2}
$$

with $\delta^{\prime}=\frac{\varepsilon_{0}}{24}$. For the last two terms we have

$$
\begin{aligned}
\sum_{b \in \mathscr{A}} & \left|\left\langle\left(1-\eta_{\varepsilon_{2}}\right) g_{b} F_{b} \eta_{\varepsilon_{2}}\right\rangle\right|+\left|\left\langle g_{b} F_{b}\left(1-\eta_{\varepsilon_{2}}\right)\right\rangle\right| \\
\leqq & \sum_{b \in \mathscr{C}} 2\left\|g_{b}^{\frac{1}{2}}(H-\bar{\lambda}+i)^{-1}\right\|^{2} \cdot\left\|(H-\bar{\lambda}+i)\left(1-\eta_{\varepsilon_{2}}(H-\bar{\lambda})\right) e^{\theta} \phi\right\| \\
& \cdot\left\|(H-\bar{\lambda}+i) e^{\theta} \phi\right\| \\
\leqq & C\left\|(H-\bar{\lambda}) e^{\theta} \phi\right\| \cdot\left(\left\|(H-\bar{\lambda}) e^{\theta} \phi\right\|+\left\|e^{\theta} \phi\right\|\right) .
\end{aligned}
$$

As in the estimates below (4.13), we obtain (in the more complicated case pertaining to Lemma 4.2)

$$
\begin{aligned}
\left\|(H-\bar{\lambda}) e^{0} \phi\right\|^{2} & \leqq 2\left\|e^{\theta}(H-\lambda) \phi\right\|^{2}+2\left\|\left(\alpha_{0}^{2}-\left(\theta^{\prime 2}-\theta^{\prime \prime}\right)|\nabla f|^{2}+2 i \theta^{\prime} A_{f}\right) e^{\theta} \phi\right\|^{2} \\
& \leqq 2\left\|e^{\theta}(H-\lambda) \phi\right\|^{2}+C\left\|\left(\langle x\rangle^{-1}+\kappa^{-1}+\delta\right) e^{\theta} \phi\right\|^{2}+4 \sup \theta^{\prime}\left\langle T_{1}\right\rangle .
\end{aligned}
$$

Here, as before, $\gamma+\left|\alpha-\alpha_{0}\right| \leqq \delta$. We choose $\delta$ small and $\kappa$ large to obtain

$$
\sum_{b \in \mathscr{A}}\left|\left\langle g_{b} F_{b}\right\rangle\right| \leqq C\left\|e^{0}(H-\lambda) \phi\right\|^{2}+2 \delta^{\prime}\left\|e^{\theta} \phi\right\|^{2}+\frac{1}{4}\left\langle T_{1}\right\rangle+\langle o(1)\rangle .
$$

The other terms in (6.3) are estimated exactly as in Sect. 4. The term $\left\langle\frac{T_{1}}{2}\right\rangle$ in (4.19) can be replaced by $\left\langle\frac{T_{1}}{4}\right\rangle$ without any change in the argument. Then

$$
\left\langle\phi, i\left[H, e^{0} A_{f} e^{0}\right] \phi\right\rangle+C\left\|e^{0}(H-\lambda) \phi\right\|^{2} \geqq \frac{\varepsilon_{0}}{12}\left\|e^{0} \phi\right\|^{2}-\left\|K e^{0} \phi\right\|^{2} .
$$

In order to use (6.4) to prove that if $(H-\lambda) \psi=0$, then $e^{\alpha\langle x\rangle} \psi \in L^{2}(X)$ for all $\alpha$ we approximate $\psi$ by a sequence $\chi_{n} \psi$ of compact support functions in $\mathscr{D}(H)$ for which (6.4) is easily seen to be valid. Here $\chi_{n}(x)=\chi_{1}(x / n), \chi_{1} \in C_{0}^{\infty}$ with $\chi_{1}=1$ for $|x| \leqq 1$. We then need to show

$$
\begin{gathered}
e^{\theta}(H-\lambda) \chi_{n} \psi \rightarrow 0, \\
\left\|p e^{\theta} \chi_{n} \psi\right\| \leqq \text { const } .
\end{gathered}
$$

Notice that Lemmas 3.1 and 3.2 remain valid. 
These facts and judicious choices of $h$ are enough to prove (6.5) from which we learn that $(H-\lambda) \psi=0$ implies $e^{\alpha\langle x\rangle} \psi \in L^{2}(X)$ for all $\alpha$. there as

To obtain a contradiction we follow the proof in Sect. 4 . We rewrite a calculation

$$
\left\|e^{\alpha E \cdot x}\left(H_{0}-\lambda\right) \phi\right\| \geqq\left\|\left(H_{0}-\bar{\lambda}+i \gamma\right) e^{\alpha E \cdot x} \phi\right\| ; \quad \phi \in C_{0}^{\infty},
$$

where $\gamma=\sqrt{2 \alpha|E|^{2}}, \bar{\lambda}=\lambda+\alpha^{2}|E|^{2}$, and where $\alpha \geqq 0$. If we let $\beta(\gamma)=\sup _{\mu \in \mathbf{R}}$ $\left\|V\left(H_{0}-\mu+i \gamma\right)^{-1}\right\|$ and choose $\gamma$ large enough so that $\beta(\gamma)<\frac{1}{2}$ we have

$$
\begin{aligned}
\left\|e^{\alpha E \cdot x}(H-\lambda) \phi\right\| & \geqq\left\|e^{\alpha E \cdot x}\left(H_{0}-\lambda\right) \phi\right\|-\left\|V e^{\alpha E \cdot x} \phi\right\| \\
& \geqq\left\|\left(H_{0}-\bar{\lambda}+i \gamma\right) e^{\alpha E \cdot x} \phi\right\|-\beta(\gamma)\left\|\left(H_{0}-\bar{\lambda}+i \gamma\right) e^{\alpha E \cdot x} \phi\right\| \\
& \geqq\left(\frac{\gamma}{2}\right)\left\|e^{\alpha E \cdot x} \phi\right\|
\end{aligned}
$$

It is clearly enough to show that (6.6) is valid for the eigenfunction $\psi$ to obtain a contradiction. But the necessary approximations have already been discussed.

Proof of Theorem 6.3. We shall use Appendix B. Absence of singular continuous spectrum and existence of the first limits follow from Proposition B.4, Corollary B.6, (B.5), and the abstract Mourre theory [M,PSS]. The remaining part of the theorem follows from existence of the first limits in conjunction with the first resolvent formula and Lemma 3.1 (cf. Sect. 5).

\section{Appendix A. Proof of Proposition 6.4}

We shall prove Proposition 6.4 under the additional condition $\Pi E \neq 0$. See the end of the appendix for the case $\Pi E=0$.

Under the above assumption the strategy of the proof is (in both cases (1) and (2)) to reduce (by a partial Fourier transform and Airy functions) to estimates of explicit kernels. We shall extensively use the notation $C$ for non-zero constants.

First we prove (1). Clearly we can assume that $\operatorname{dim} X=3$ and (by a scaling) that $|E|=1$. By a suitable choice of coordinates $x=\left(x_{\perp}, z\right) \in \mathbf{R}^{2} \times \mathbf{R}$ it is then enough to bound each of the following expressions independently of $\lambda$ and $\gamma$ :

$$
\begin{aligned}
& D_{1}:=|\gamma|^{\frac{1}{2}}\left\|\frac{1}{\langle z\rangle}(-\Delta+z-\lambda-i \gamma)^{-1}\right\|, \\
& D_{2}:=|\gamma|^{\frac{1}{2}}\left\|\frac{1}{|x|} F(|z|<1)(-\Delta+z-\lambda-i \gamma)^{-1}\right\| .
\end{aligned}
$$

To bound $D_{1}$ we conjugate by the partial Fourier transform with respect to $x_{\perp}$, denoted $F_{\perp}$, to reduce the problem to a one-dimensional estimate. It is enough to bound

$$
\tilde{D}_{1}:=|\gamma|^{\frac{1}{2}}\left\|\frac{1}{\langle z\rangle}\left(h_{0}-\lambda-i \gamma\right)^{-1}\right\| ; \quad h_{0}=-\frac{d^{2}}{d z^{2}}+z,
$$

independently of $\lambda$ and $\gamma$. For that we use the fact that the one-dimensional Stark Hamiltonian $h_{0}$ is diagonalized by the Airy function $\operatorname{Ai}(\cdot)$. Explicitly the delta 
function $\delta\left(h_{0}\right)=(2 \pi i)^{-1} \lim _{\varepsilon \downarrow 0}\left(\left(h_{0}-i \varepsilon\right)^{-1}-\left(h_{0}+i \varepsilon\right)^{-1}\right)$ has the kernel

$$
\delta\left(h_{0}\right)\left(z, z^{\prime}\right)=\operatorname{CAi}(z) \operatorname{Ai}\left(z^{\prime}\right) \text {. }
$$

By (A.1) and a translation property of $h_{0}$ we obtain the following expression for the delta function at any real energy $\mu$,

$$
\delta\left(h_{0}-\mu\right)\left(z, z^{\prime}\right)=C A i(z-\mu) \operatorname{Ai}\left(z^{\prime}-\mu\right) .
$$

We shall only need (for the proof of (1)) the bound (cf. [AS, 10.4.59-60])

$$
|A i(z)| \leqq C\langle z\rangle^{-\frac{1}{4}} ; \quad z \in \mathbf{R}
$$

By a standard application of the spectral theorem we can represent the square of $\tilde{D}_{1}$ as the norm of an integral (with respect to $\mu$ ) of the function

$$
\frac{\gamma}{(\mu-\lambda)^{2}+\gamma^{2}}
$$

times the operator whose kernel is given by (A.2) times the factor $1 /\langle z\rangle\left\langle z^{\prime}\right\rangle$. From this representation we obtain readily by pulling the norm through the integral that it is enough to bound the rank-one operator given by the kernel

$$
\frac{1}{\langle z\rangle} \operatorname{Ai}(z-\mu) \operatorname{Ai}\left(z^{\prime}-\mu\right) \frac{1}{\left\langle z^{\prime}\right\rangle}
$$

independently of $\mu \in \mathbf{R}$. But this is easy by the Hilbert Schmidt criterion and (A.3).

It remains to bound $D_{2}$. For that we shall use the following expression for the partial Fourier transform of the Coulomb potential (given for any $z \in \mathbf{R}$ ):

$$
F_{\perp}\left(\frac{1}{|x|}\right)(\eta)=C \frac{e^{-|z||\eta|}}{|\eta|} ; \quad \eta \in \mathbf{R}^{2} .
$$

We proceed similarly as before. The multiplication operator given by the Coulomb potential goes into convolution (with respect to $\eta$ ) by the function (A.4). Then the square of $D_{2}$ is represented as the norm of an integral of an operator valued function involving the one-dimensional delta function (A.2). Explicitly we shall complete the proof by bounding (uniformly in $\mu$ ) the norm of the operator $K_{\mu}$ given by the kernel

$$
\begin{aligned}
K_{\mu}\left(z, \eta ; z^{\prime}, \eta^{\prime}\right)= & \int_{\mathbf{R}^{2}} F(|z|<1) \frac{e^{-|z||\eta-\bar{\eta}|}}{|\eta-\bar{\eta}|} A i\left(z+\bar{\eta}^{2}-\mu\right) \\
& \times \operatorname{Ai}\left(z^{\prime}+\bar{\eta}^{2}-\mu\right) \frac{e^{-\left|z^{\prime}\right|\left|\bar{\eta}-\eta^{\prime}\right|}}{\left|\bar{\eta}-\eta^{\prime}\right|} F\left(\left|z^{\prime}\right|<1\right) d \bar{\eta} .
\end{aligned}
$$

Clearly by (A.3) it thus suffices to bound the operator $G_{\mu}$ given by the kernel

$$
G_{\mu}\left(z, \eta ; z^{\prime}, \eta^{\prime}\right)=\int_{\mathbf{R}^{2}}\left\langle\bar{\eta}^{2}-\mu\right\rangle^{-\frac{1}{2}} \frac{e^{-|z||\eta-\bar{\eta}|}}{|\eta-\bar{\eta}|} \frac{e^{-\left|z^{\prime}\right|\left|\bar{\eta}-\eta^{\prime}\right|}}{\left|\bar{\eta}-\eta^{\prime}\right|} d \bar{\eta} .
$$

Since

$$
\sup _{\bar{\eta} \in \mathbf{R}^{2}, \mu \leqq 0}\left\langle\bar{\eta}^{2}-\mu\right\rangle^{-\frac{1}{2}}\left\langle\bar{\eta}^{2}-1\right\rangle^{\frac{1}{2}}<\infty
$$


we only need to bound uniformly in $\mu>0$. For that we replace the factor $\left\langle\bar{\eta}^{2}-\mu\right\rangle^{-\frac{1}{2}}$ in the integrand by the (larger) expression $\left|\bar{\eta}^{2}-\mu\right|^{-\frac{1}{2}}$. In this way we obtain a family of unitarily equivalent operators as follows by the scaling $z \rightarrow \mu^{-\frac{1}{2}} z, \eta \rightarrow \mu^{\frac{1}{2}} \eta$. Hence it only remains to show boundedness of the operator $G$ given by the kernel

$$
G\left(z, \eta ; z^{\prime}, \eta^{\prime}\right)=\int_{\mathbf{R}^{2}}\left|\bar{\eta}^{2}-\mu\right|^{-\frac{1}{2}} \frac{e^{-|z||\eta-\bar{\eta}|}}{|\eta-\bar{\eta}|} \frac{e^{-\left|z^{\prime}\right|\left|\bar{\eta}-\eta^{\prime}\right|}}{\left|\bar{\eta}-\eta^{\prime}\right|} d \bar{\eta} .
$$

For that we choose real $\alpha$ and $\beta$ such that

$$
\frac{1}{2}>\beta>\alpha>0 \text {. }
$$

Then by the Schur test [HS, Theorem 5.2] $G$ is bounded if

$$
\sup _{(z, \eta) \in \mathbf{R} \times \mathbf{R}^{2}}|z|^{\alpha}\langle\eta\rangle^{\beta} \iint G\left(z, \eta ; z^{\prime}, \eta^{\prime}\right)\left|z^{\prime}\right|^{-\alpha}\left\langle\eta^{\prime}\right\rangle^{-\beta} d z^{\prime} d \eta^{\prime}<\infty .
$$

Since

$$
\sup _{z \in \mathbf{R}}|z|^{\alpha} e^{-|z||\eta-\bar{\eta}|}=C|\eta-\bar{\eta}|^{-\alpha}
$$

and (possibly with another constant)

$$
\int_{-\infty}^{\infty} e^{-\left|z^{\prime}\right|\left|\bar{\eta}-\eta^{\prime}\right|}\left|z^{\prime}\right|^{-\alpha} d z^{\prime}=C\left|\bar{\eta}-\eta^{\prime}\right|^{\alpha-1}
$$

we are led to showing finiteness of the following expression:

$$
\sup _{\eta \in \mathbf{R}^{2}}\langle\eta\rangle^{\beta} \iint\left|\bar{\eta}^{2}-1\right|^{-\frac{1}{2}}|\eta-\bar{\eta}|^{-1-\alpha}\left|\bar{\eta}-\eta^{\prime}\right|^{\alpha-2}\left\langle\eta^{\prime}\right\rangle^{-\beta} d \bar{\eta} d \eta^{\prime} .
$$

Obviously finiteness is a consequence of the following two bounds which in turn follow readily from (A.5) and Lemmas A.1 and A.2 given below.

$$
\begin{gathered}
\int\left|\bar{\eta}-\eta^{\prime}\right|^{\alpha-2}\left\langle\eta^{\prime}\right\rangle^{-\beta} d \eta^{\prime} \leqq C\langle\bar{\eta}\rangle^{\alpha-\beta}, \\
\int\left|\bar{\eta}^{2}-1\right|^{-\frac{1}{2}}|\eta-\bar{\eta}|^{-1-\alpha}\langle\bar{\eta}\rangle^{\alpha-\beta} d \bar{\eta} \leqq C\langle\eta\rangle^{-\beta} .
\end{gathered}
$$

As it holds for (A.6) and (A.7) the following statements involve only twodimensional variables.

Lemma A.1. Suppose $1<k<2$ and $0<l<2-k$. Then

$$
\sup _{|\bar{\eta}|<2} \int_{\left|\eta^{\prime}\right|<2}\left|\bar{\eta}-\eta^{\prime}\right|^{-k}\left|\eta^{\prime 2}-1\right|^{-l} d \eta^{\prime}<\infty .
$$

Proof. Only the contribution to the integral from the region $\frac{1}{2}<\left|\eta^{\prime}\right|<2$ needs to be considered. Changing to polar coordinates,

$$
\eta^{\prime}=(r \cos \theta, r \sin \theta), \quad \bar{\eta}=(s, 0),
$$


we have the bound

$$
|\bar{\eta}-\eta| \geqq C\left((r-s)^{2}+\theta^{2}\right)^{\frac{1}{2}}
$$

But

$$
\int_{\frac{1}{2}}^{2} d r \frac{r}{\left|r^{2}-1\right|^{l}} \int_{-\pi}^{\pi} d \theta\left((r-s)^{2}+\theta^{2}\right)^{-\frac{k}{2}} \leqq C \int_{\frac{1}{2}}^{2} \frac{r}{\left|r^{2}-1\right|^{l}}|r-s|^{1-k} d r,
$$

which by the Hölder inequality can be estimated independently of $s<2$.

Lemma A.2. Suppose $0<k, l<2$ and $2<k+l$. Then

$$
\int\left|\bar{\eta}-\eta^{\prime}\right|^{-k}\left\langle\eta^{\prime}\right\rangle^{-l} d \eta^{\prime} \leqq C\langle\bar{\eta}\rangle^{2-k-l}
$$

Proof. We look separately at the regions

$$
\begin{aligned}
& R_{1}:\left|\bar{\eta}-\eta^{\prime}\right|<\frac{1}{2}|\bar{\eta}|, \\
& R_{2}:\left|\bar{\eta}-\eta^{\prime}\right| \geqq \frac{1}{2}|\bar{\eta}|,\left|\eta^{\prime}\right| \leqq 2|\bar{\eta}|, \\
& R_{3}:\left|\eta^{\prime}\right|>2|\bar{\eta}| .
\end{aligned}
$$

In case of the region $R_{1},\left|\eta^{\prime}\right|>\frac{1}{2}|\bar{\eta}|$, which permits us to estimate

$$
\begin{aligned}
\int_{R_{1}}\left|\bar{\eta}-\eta^{\prime}\right|^{-k}\left\langle\eta^{\prime}\right\rangle^{-l} d \eta^{\prime} & \leqq 4\langle\bar{\eta}\rangle^{-l} \int_{R_{1}}\left|\bar{\eta}-\eta^{\prime}\right|^{-k} d \eta^{\prime} \\
& =C\langle\bar{\eta}\rangle^{-l}|\bar{\eta}|^{2-k} \leqq C\langle\bar{\eta}\rangle^{2-k-l} .
\end{aligned}
$$

In case of the region $R_{2}$,

$$
\begin{aligned}
\int_{R_{2}}\left|\bar{\eta}-\eta^{\prime}\right|^{-k}\left\langle\eta^{\prime}\right\rangle^{-l} d \eta^{\prime} & \leqq C|\bar{\eta}|^{-k} \int_{R_{2}}\left\langle\eta^{\prime}\right\rangle^{-l} d \eta^{\prime} \\
& \leqq C|\bar{\eta}|^{-k} \int_{\left|\eta^{\prime}\right| \leqq 2|\bar{\eta}|}\left|\eta^{\prime}\right|^{-l} d \eta^{\prime}=C|\bar{\eta}|^{2-k-l} .
\end{aligned}
$$

Since the estimate of Lemma A.2 is obvious for $\bar{\eta}$ in a compact set we conclude that

$$
\int_{R_{2}}\left|\bar{\eta}-\eta^{\prime}\right|^{-k}\left\langle\eta^{\prime}\right\rangle^{-l} d \eta^{\prime} \leqq C\langle\bar{\eta}\rangle^{2-k-l}
$$

As for the region $R_{3}$ we estimate

$$
\begin{aligned}
\int_{R_{3}}\left|\bar{\eta}-\eta^{\prime}\right|^{-k}\left\langle\eta^{\prime}\right\rangle^{-l} d \eta^{\prime} & \leqq 2^{k} \int_{R_{3}}\left|\eta^{\prime}\right|^{-k}\left\langle\eta^{\prime}\right\rangle^{-l} d \eta^{\prime} \\
& \leqq C|\bar{\eta}|^{2-k-l} .
\end{aligned}
$$

So (again) we obtain

$$
\int_{R_{3}}\left|\bar{\eta}-\eta^{\prime}\right|^{-k}\left\langle\eta^{\prime}\right\rangle^{-l} d \eta^{\prime} \leqq C\langle\bar{\eta}\rangle^{2-k-l}
$$

We have proved (1) (for $\Pi E \neq 0$ ). 
As for the second statement (2) of Proposition 6.4 we proceed by assuming finiteness of the quantity

$$
D:=\sup _{\lambda \in \mathbf{R}}\left\|V(x)(-\Delta+z-\lambda-i)^{-1}\right\|
$$

and then obtaining a contradiction. As in the proof of (1) we have chosen coordinates $x=\left(x_{\perp}, z\right) \in \mathbf{R}^{(d-1)} \times \mathbf{R}$ for $\Pi X$. The corresponding $d$-dimensional momentum operator decomposes accordingly, $p=\left(p_{\perp}, p_{d}\right)$. We introduce for $\lambda>0$,

$$
K_{\lambda}=\frac{1}{|x|} F\left(|z|<\lambda^{-\frac{1}{2}}\right)(-\Delta+z-\lambda-i)^{-1} F\left(z<-\lambda^{\frac{1}{2}}\right) F\left(p_{\perp}^{2}<\frac{\lambda}{2}\right) .
$$

As a family of bounded operators on $L^{2}\left(\mathbf{R}^{d}\right)$ we shall prove the following result from which in particular (2) will follow readily. Let $K: L^{2}\left(\mathbf{R}_{\eta^{\prime}}^{(d-1)}\right) \rightarrow L^{2}\left(\mathbf{R}_{z} \times\right.$ $\mathbf{R}_{\eta}^{(d-1)}$ ) be the operator given by the kernel

$$
K\left(z, \eta ; \eta^{\prime}\right)=F(|z|<1) F\left(\eta^{\prime 2}<\frac{1}{2}\right) f_{z}\left(\eta-\eta^{\prime}\right) e^{l z\left(1-\eta^{\prime 2}\right)^{\frac{1}{2}}}\left(1-\eta^{\prime 2}\right)^{-\frac{1}{4}} e^{-\frac{1}{2}\left(1-\eta^{\prime 2}\right)^{-\frac{1}{2}}}
$$

where $f_{z}(\eta)$ denotes the $(d-1)$-dimensional Fourier transform of the Coulomb potential $|x|^{-1}$ (with respect to $x_{\perp}$ for fixed $z$ ). We notice that $K$ is bounded as can easily be proved using the bound (cf. (A.4))

$$
\left|f_{z}(\eta)\right| \leqq C \frac{e^{-\frac{|z||\eta|}{2}}}{|\eta|^{d-2}}
$$

Then

Lemma A.3. There exist partial isometries $U_{\lambda}$ and $V_{\lambda}$ and a (non-zero) constant C such that

$$
\mathrm{w}-\lim _{\lambda \rightarrow \infty} U_{\lambda} K_{\lambda} V_{\lambda}=C K .
$$

Proof. First we conjugate by the unitary operator implemented by the scaling $x \rightarrow$ $\lambda^{-\frac{1}{2}} x$. Then we conjugate by the partial Fourier transform appearing also in the proof of (1) to reduce the $d$-dimensional resolvent to a resolvent of the one-dimensional Stark operator $h_{0}$ which in turn is given by an explicit kernel in terms of the Airy function. Using this expression we obtain that $K_{\lambda}$ is given up to unitary equivalence by the kernel

$$
\begin{gathered}
\tilde{K}_{\lambda}\left(z, \eta ; z^{\prime}, \eta^{\prime}\right)= \\
C F(|z|<1) F\left(z^{\prime}<-\lambda\right) F\left(\eta^{\prime 2}<\frac{1}{2}\right) \\
\times f_{z}\left(\eta-\eta^{\prime}\right) \operatorname{Ai}\left(\lambda^{-\frac{1}{2}} z-\mu(\lambda)\right) \operatorname{Ai}\left(e^{\frac{2 \pi}{3} l}\left(\lambda^{-\frac{1}{2}} z^{\prime}-\mu(\lambda)\right)\right) ; \\
\mu(\lambda)=\lambda-\lambda \eta^{\prime 2}+i .
\end{gathered}
$$

By the asymptotics of the Airy function [AS, (10.4.59)]

$$
\left(\int_{-\infty}^{-\lambda}\left|\operatorname{Ai}\left(e^{\frac{2 \pi}{3} i}\left(\lambda^{-\frac{1}{2}} z^{\prime}-\mu(\lambda)\right)\right)\right|^{2} d z^{\prime}\right)^{\frac{1}{2}} \sim C \lambda^{\frac{1}{4}} e^{-\left(\lambda-\lambda \eta^{\prime 2}+\lambda^{\frac{1}{2}}\right)^{\frac{1}{2}}} \quad \text { for } \quad \lambda \rightarrow \infty,
$$


uniformly in $\eta^{\prime 2}<\frac{1}{2}$. (This means that the quotient of the two expressions has a non-zero limit as $\lambda \rightarrow \infty$.) Consequently it suffices to look at the kernel

$$
\begin{aligned}
G_{\lambda}\left(z, \eta ; \eta^{\prime}\right)= & C F(|z|<1) F\left(\eta^{\prime 2}<\frac{1}{2}\right) \\
& \times f_{z}\left(\eta-\eta^{\prime}\right) A i\left(\lambda^{-\frac{1}{2}} z-\mu(\lambda)\right) \lambda^{\frac{1}{4}} e^{-\left(\lambda-\lambda \eta^{\prime 2}+\lambda^{\frac{1}{2}}\right)^{\frac{1}{2}}} .
\end{aligned}
$$

As for the remaining factor given in terms of the Airy function we use the asymptotics [AS, (10.4.60)]

$$
\begin{aligned}
\operatorname{Ai}\left(\lambda^{-\frac{1}{2}} z-\mu(\lambda)\right) \sim & C \lambda^{-\frac{1}{4}}\left(1-\eta^{2}\right)^{-\frac{1}{4}} e^{\lambda^{\frac{1}{2}}\left(1-\eta^{\prime 2}\right)^{\frac{1}{2}}} \\
& \times e^{-l \frac{2}{3} \lambda^{\frac{3}{2}}\left(1-\eta^{\prime 2}\right)^{\frac{3}{2}}} e^{l z\left(1-\eta^{\prime 2}\right)^{\frac{1}{2}}} \text { for } \lambda \rightarrow \infty,
\end{aligned}
$$

the statement being uniform in $|z|<1$ and $\eta^{\prime 2}<\frac{1}{2}$.

The leading contribution to the asymptotics of $G_{i}$ is obtained by inserting (A.9) into (A.8). The resulting kernel is a product of a factor independent of $\lambda$ and the product

$$
\begin{aligned}
& e^{\lambda^{\frac{1}{2}}\left(1-\eta^{\prime 2}\right)^{\frac{1}{2}}} e^{-\left(\lambda-\lambda \eta^{\prime 2}+\lambda^{\frac{1}{2}}\right)^{\frac{1}{2}}} e^{-i \frac{2}{3} \lambda^{\frac{3}{2}}\left(1-\eta^{2}\right)^{\frac{3}{2}}} \\
& \quad \sim e^{-\frac{1}{2}\left(1-\eta^{\prime 2}\right)^{-\frac{1}{2}}} e^{-i \frac{2}{3} \lambda^{\frac{3}{2}}\left(1-\eta^{\prime 2}\right)^{\frac{3}{2}}} \text { for } \lambda \rightarrow \infty
\end{aligned}
$$

(uniformly in $\eta^{2}<\frac{1}{2}$ ). Clearly multiplication by the latter $\lambda$-dependent factor can be expressed in terms of an isometry.

To complete the proof of (2) we write for any $\varepsilon>0$,

$$
\frac{1}{|x|} \leqq \varepsilon|V(x)|+C_{\varepsilon} F\left(|x|<R_{\varepsilon}\right)+\varepsilon
$$

for suitable positive constants $C_{\varepsilon}$ and $R_{\varepsilon}$ and estimate

$$
\begin{aligned}
& \liminf _{\lambda \rightarrow \infty}\left\|\frac{1}{|x|}(-\Delta+z-\lambda-i)^{-1}\right\| \\
& \quad \leqq \varepsilon D+C_{\varepsilon} \liminf _{\lambda \rightarrow \infty}\left\|F\left(|x|<R_{\varepsilon}\right)(-\Delta+z-\lambda-i)^{-1}\right\|+\varepsilon .
\end{aligned}
$$

By Lemma 3.3 the right-hand side is equal to $\varepsilon D+\varepsilon$ and since $\varepsilon$ is arbitrary we conclude that

$$
\liminf _{\lambda \rightarrow \infty}\left\|\frac{1}{|x|}(-\Delta+z-\lambda-i)^{-1}\right\|=0
$$

(using the assumption $D<\infty$ ).

Obviously (A.10) contradicts Lemma A.3. So $D=\infty$.

Now we consider the case $\Pi E=0$. For (1) we can assume $\Pi=I$. Then since we have strong resolvent convergence as $E \rightarrow 0$ the bound follows from the case $E \neq 0$ by a simple scaling argument. (Alternatively, see the remark after the proof of Lemma 6.5.) 
For (2) we note that (by another scaling)

$$
\begin{aligned}
\lim _{\lambda \rightarrow \infty}\left\||x|^{-1}\left(p^{2}-\lambda-i\right)^{-1}\right\|^{2} & =\lim _{\lambda \rightarrow \infty}\left\||x|^{-1} \operatorname{Im}\left(\left(p^{2}-1-\frac{i}{\lambda}\right)^{-1}\right)|x|^{-1}\right\| \\
& =\pi\left\||x|^{-1} \delta\left(p^{2}-1\right)|x|^{-1}\right\| .
\end{aligned}
$$

Since the latter expression is non-zero we can now proceed exactly as above.

\section{Appendix B. A Mourre Estimate for Coulomb Singularities}

The potentials considered in this appendix are assumed to satisfy Conditions 6.1. We write $V$ on the form

$$
V=W+V_{\text {sing }},
$$

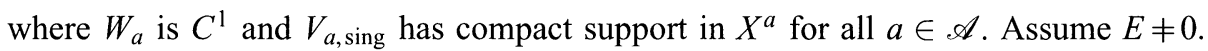

We aim at constructing an observable that commutes with the singular part of the potential and such that it locally has a positive commutator with the Stark Hamiltonian. The observable will be of the form

$$
A=\frac{1}{2}(E(x) \cdot p+p \cdot E(x)) .
$$

Our choice of the vector field $E(x)$ might seem somewhat complicated so we have devoted the beginning of this section to motivate it. The vector field $E(x)$ can be viewed as a modification of a vector field constructed in [T3].

When we write for operators $A$ and $B, A \sim B$, we mean that locally in energy the difference $A-B$ can be made arbitrarily small by the use of Corollary 6.7 . Furthermore we will denote by $M(\cdot)$ bounded functions $x \rightarrow M(x)$ with values in the selfadjoint operators on $X$.

To begin with we assume that $E_{a} \neq 0$ for all $a \neq a_{\max }$. (This is true for "atoms.") A natural first choice of $E(x)$ is

$$
E(x)=\sum_{a \neq a_{\max }} j_{a} \chi(|x|>R) E_{a},
$$

where $j_{a}$ is defined by (3.2-3). We compute

$$
\begin{aligned}
i[H, A] & \geqq \chi(|x|>R) \min _{a \neq a_{\max }}\left(\left|E_{a}\right|^{2}\right)+i\left[p^{2}, A\right]+i[V, A] \\
& \sim \min _{a \neq a_{\max }}\left(\left|E_{a}\right|^{2}\right)+p^{t}\langle x\rangle^{-1} M(x) p+i[V, A] .
\end{aligned}
$$

Since we can choose $R$ large enough such that $i[V, A]=i[W, A]$ is small we are almost done. The problem is the second term. In order to handle this term we add to $E_{a}$ the term $K \hat{x}, K>0, \hat{x}=\frac{x}{\langle x\rangle}$, and compute

$$
\begin{aligned}
i\left[H, \sum_{a \neq a_{\max }} j_{a} \chi(|x|>R) K \hat{x} \cdot p\right] & \sim p^{t} K\left\{\langle x\rangle^{-1} I+2 \hat{x}^{\prime}\right\} p-\chi(|x|>R) K \hat{x} \cdot \nabla V \\
& \geqq p^{t} K\langle x\rangle^{-1} I p-\chi(|x|>R) K \hat{x} \cdot \nabla V
\end{aligned}
$$


where $I$ denotes the identity. Here we used that the derivative $\hat{x}^{\prime}$ is positive and that $E \cdot \hat{x} \sim p^{t}\langle x\rangle^{-1} I p$. By choosing $K$ large enough one can estimate $K I+M(x) \geqq$ 0 . Our remaining problem is the last term. If we instead of $\hat{x}=\nabla\langle x\rangle$ choose the gradient of Derezinski's approximation to $\langle x\rangle$ which satisfies (D1-4) below, we obtain $\chi(|x|>R) \nabla r \cdot \nabla V=\chi(|x|>R) \nabla r \cdot \nabla W$ which is small for $R$ large.

The vector field we get under the above assumption on $E_{a}$ is

$$
E(x)=\sum_{a \neq a_{\max }} j_{a} \chi(|x|>R)\left(E_{a}+K \nabla r\right),
$$

where $K$ and $R$ are chosen such that we obtain the desired properties. A similar vector field (though constructed as a gradient field) was considered in [Sk].

In the general case where $E_{a}$ can be zero we will need the concept of strings and partitions of unity with respect to subsystems as introduced below. The construction of the vector field is essentially the above one with alterations needed to suit the general situation.

We will need partitions of unity $j_{b}^{a}, b \subsetneq a$ defined for all subsystem $a \in \mathscr{A}$. The partitions $j_{b}^{a}: X^{a} \rightarrow[0,1]$ are smooth and homogeneous of degree 0 outside a compact set. Moreover we assume that

$$
\sum_{b \subsetneq a} j_{b}^{a}\left(x^{a}\right)=1
$$

For any pair $b, c \subset a$ such that $b \neq a$ and $c \not \subset b$ there exists $C>0$ such that

$$
\left|x^{c}\right| \geqq C\left|x^{a}\right|, \quad \text { on } \operatorname{supp} j_{b}^{a} \text {. }
$$

We will use Derezinski's smooth approximation to $|x|$, [De, Proposition 4.4]. For all $a \in \mathscr{A}$ we have a smooth real function $r^{a}$ on $X^{a}$ satisfying

(D1) $r^{a}$ is convex.

(D2) For all $b \subset a$ we have

$$
\nabla r^{a}(x) \cdot \nabla V_{b, \text { sing }}\left(x^{b}\right)=0 .
$$

(D3) For all $b \subset a$ there exists $C_{a, b}>0$ such that

$$
\nabla r^{a}\left(x^{a}\right) \cdot \nabla W_{b}\left(x^{b}\right) \neq 0 \Rightarrow\left|x^{b}\right| \geqq C_{a, b}\left|x^{a}\right| .
$$

(D4) $\forall \alpha \exists C_{\alpha}>0$ such that $\left|\partial_{x}^{\alpha}\left(r^{a}\left(x^{a}\right)-\left\langle x^{a}\right\rangle\right)\right| \leqq C_{\alpha}\left\langle x^{a}\right\rangle^{-1}$.

We can without loss of generality assume that there exist an $a^{\prime} \in \mathscr{A}$ such that $X^{a^{\prime}}=\operatorname{span}\{E\}$ (if not we can add subspaces to the family with zero potentials).

We define a string $\bar{a}=\left\{a_{0}, \ldots, a_{n(\bar{a})}\right\}$ to be a sequence of $n(\bar{a})+1$ elements from $\mathscr{A}$ satisfying

(S1) $a_{\max }=a_{0} \supsetneq a_{1} \supsetneq \cdots \supsetneq a_{n(\bar{a})}$.

(S2) For $i<n(\bar{a})$ we have $E_{a_{l}}=0$.

(S3) $E_{a_{n(\bar{a})}}=0$ implies $a_{n(\bar{a})}=a^{\prime}$.

Denote the set of strings, which is finite, by $S(\mathscr{A})$. For $k \geqq 1$ we define the set $S_{k}(\mathscr{A})=\{\bar{a} \in S(\mathscr{A}) \mid n(\bar{a}) \geqq k\}$ and an equivalence relation on $S_{k}(\mathscr{A})$ by

$$
\bar{a} \sim \bar{b} \Leftrightarrow a_{l}=b_{l}, \quad 0 \leqq i \leqq k-1 .
$$


We define partitions

$$
j_{\bar{a}}(x)=\prod_{1 \leqq k \leqq n(\bar{a})} j_{a_{k}}^{a_{k-1}}\left(x^{a_{k-1}}\right) .
$$

For notational convenience we define for $1 \leqq k \leqq n(\bar{a})$,

$$
\vec{j}_{\bar{a}, k}=\left(\prod_{1 \leqq i<k} j_{a_{l}}^{a_{l-1}}\right)\left(\nabla j_{a_{k}}^{a_{k-1}}\right)\left(\prod_{k<i \leqq n(\bar{a})} j_{a_{l}}^{a_{l-1}}\right)
$$

such that we have

$$
\nabla j_{\bar{a}}=\sum_{1 \leqq k \leqq n(\bar{a})} \vec{j}_{\bar{a}, k}
$$

Since strings can be viewed as being obtained inductively starting from $a_{\max }$ by extending the sequence with smaller elements terminating the procedure when either $E_{a_{n}} \neq 0$ or when $a_{n}=a^{\prime}$ we obtain the following lemma

Lemma B.1. Let $k \geqq 1$ and $\bar{b} \in S_{k}(\mathscr{A})$ be given. We have

$$
\begin{aligned}
& \sum_{\bar{a} \in S(\mathscr{A})} j_{\bar{a}}=1 . \\
& \sum_{\substack{\bar{a} \in S_{k}(\mathscr{A}) \\
\bar{a} \sim \bar{b}}} \vec{j}_{\bar{a}, k}=0 . \\
& \sum_{\substack{\bar{a} \in S_{k}(\cdot \mathscr{d}) \\
\bar{a} \sim \bar{b}}} j_{\bar{a}}=j_{b_{1}}^{b_{0}} \ldots j_{b_{k-1}}^{b_{k-2}} .
\end{aligned}
$$

Notice that $N \geqq 1+\max _{\bar{a} \in S(\mathscr{A})} n(\bar{a})$, where $N$ is the "number of particles" in the system as defined in Sect. 2 .

We will need the following two results which can be proved in the same way as for Lemma 3.1,

$$
\langle z\rangle^{-\frac{1}{2}} \vec{q}_{0} \cdot p(H+i)^{-1} \in \mathscr{B}\left(L^{2}(X)\right),
$$

and

$$
\langle z\rangle^{-1} p^{2}(H+i)^{-1} \in \mathscr{B}\left(L^{2}(X)\right),
$$

where $\vec{q}_{0}$ is any bounded continuous function and $z=x^{a^{\prime}}=|\omega\rangle\langle\omega \mid x\rangle, \omega=\frac{E}{|E|}$.

We aim at producing a conjugate operator that commutes with $V_{\text {sing }}$.

We define a function $E: S(\mathscr{A}) \rightarrow X$ by

$$
E(\bar{a})= \begin{cases}E_{a_{n(\bar{a})}}, & E_{a_{n(\bar{a})}} \neq 0 \\ E, & E_{a_{n(\bar{a})}}=0\end{cases}
$$

Let

$$
e=\min _{\substack{a \in \mathscr{\alpha} \\ E_{a} \neq 0}}\left|E_{a}\right|^{2}
$$


Lemma B.2. Let $K \geqq 1, \bar{a} \in S(\mathscr{A})$ and $b \neq a_{\min }$ be given. There exists $R=$ $R(K, \bar{a}, b)>0$ such that we have

(1) $F(|z| \geqq R) j_{\bar{a}} E_{K}(\bar{a}, x) \cdot \nabla V_{b, \text { sing }}\left(x^{b}\right)=0$.

(2) $\left|F(|z| \geqq R) j_{\bar{a}} E_{K}(\bar{a}, x) \cdot \nabla W_{b}\left(x^{b}\right)\right| \leqq \frac{e}{4|. /||S(.,)|}$,

where

$$
E_{K}(\bar{a}, x)=E(\bar{a})+\nabla r_{K}^{\bar{a}}(x)
$$

and

$$
r_{K}^{\bar{a}}(x)=\sum_{1 \leqq l \leqq n(\bar{a})} K^{N-l} r^{a_{l-1}}\left(x^{a_{l-1}}\right)
$$

Proof. First consider the case $b \subset a_{n(\bar{a})}$.

If $E_{a_{n(\bar{a})}}=0$ we have $a_{n(\bar{a})}=a^{\prime}=b$ by (S3). Thus we can by Conditions 6.1 choose $R$ large enough such that (1) and (2) hold.

If $E_{a_{n(\bar{a})}} \neq 0$ we have $E(\bar{a}) \cdot \nabla V_{b \text {,sing }}\left(x^{b}\right)=E(\bar{a}) \cdot \nabla W_{b}\left(x^{b}\right)=0$. (D2) assures that $\nabla r_{K}^{\bar{a}} \cdot \nabla V_{b \text {, sing }}=0$. The properties (S1-2) implies that $a^{\prime}, b \subset a_{n(\bar{a})-1}$. This observation together with (D3) assures that (2) holds by choosing $R$ large enough.

If $b \not \subset a_{n(\bar{a})}$ there exists $1 \leqq k \leqq n(\bar{a})$ such that $b \subset a_{k-1}$ and $b \not \subset a_{k}$. Since $|z|>R \Rightarrow\left|x^{a_{k-1}}\right|>R$, by (S2), we can by (B.2) choose $R$ large enough such that on the support of

$$
j_{\bar{a}}=\left(\prod_{l \leqq l<k-1} j_{a_{l}}^{a_{l-1}}\right) j_{a_{k}}^{a_{k-1}}\left(\prod_{k<l \leqq n(\bar{a})} j_{a_{l}}^{a_{l-1}}\right)
$$

we have forced $x^{b}$ out of $\operatorname{supp}\left(V_{b, \text { sing }}\right)$ and out where $\nabla W_{b}$ is sufficiently small. (Notice that $\nabla r^{a}$ is bounded by (D4).)

For any $K \geqq 1$ we define the vector field

$$
E(x)=E(x, K)=\chi(|z|>\bar{R}) \sum_{\bar{a} \in S(, \mathcal{d})} j_{\bar{a}}(x) E_{K}(\bar{a}, x),
$$

where $\bar{R}=\bar{R}(K)=\max _{\bar{a} \in S(\mathscr{c}), b \neq a_{\min }} R(K, \bar{a}, b)$.

We thus obtain the following result

Lemma B.3. For any $K \geqq 1$ we have

(1) $E(x) \cdot \nabla V_{\text {sing }}=0$.

(2) $|E(x) \cdot \nabla W| \leqq \frac{e}{4}$.

By the construction we notice that

$$
\forall \alpha \exists C_{\alpha}>0: \quad\left|\partial_{x}^{\alpha} E(x)\right| \leqq C_{\alpha}\langle z\rangle^{-\min (1,|\alpha|)} .
$$

We wish to consider the observable

$$
A=A(K)=\frac{1}{2}(E(x) \cdot p+p \cdot E(x)),
$$

which by (B.5) is essentially selfadjoint on $C_{0}^{\infty}(X)$. 
Prosposition B.4 (Mourre estimate). Let $\mathscr{K} \subset \mathbb{R}$ be a compact subset. There exist $\varepsilon>0$ and $K \geqq 1$ such that for all $\lambda \in \mathscr{K}$ we have

$$
\eta_{\varepsilon}(H-\lambda) i[H, A] \eta_{\varepsilon}(H-\lambda) \geqq \frac{e}{2} \eta_{\varepsilon}^{2}(H-\lambda) .
$$

Remark. By $[H, A]$ above we mean the extension to $\mathscr{D}(H)$ of the corresponding commutator defined as a form on $C_{0}^{\infty}(X)$. Since we will use the proposition (in Sect. 6) together with the abstract Mourre theory [M] we notice that this commutator equally well can be understood as the extension from $\mathscr{D}(H) \cap \mathscr{D}(A)$. This follows from an application of (B.5) in conjunction with [M, Proposition II.1].

Proof. We write

$$
i[H, A]=i\left[p^{2}, A\right]+i[-E \cdot x, A]+i[V, A] .
$$

We compute the three commutators using Lemma B.3 (1),

$$
\begin{aligned}
i\left[p^{2}, A\right]= & p^{t}\left\{E^{\prime}(x)+E^{\prime}(x)^{t}\right\} p-\Delta(\nabla \cdot E(x)), \\
& i[-E \cdot x, A]=E(x) \cdot E, \\
& i[V, A]=-E(x) \cdot \nabla W .
\end{aligned}
$$

Notice that by (D1) the second derivative of $r_{K}^{\bar{a}}$ is positive and since $E(\bar{a})$ does not depend on $x$ we have positivity of the matrix $E_{K}^{\prime}(\bar{a}, x)$. Using this observation together with (D4) we obtain

$$
i\left[p^{2}, A\right] \geqq p^{t} \sum_{\bar{a} \in S(\mathscr{A})} 2 \operatorname{Re}\left\{\nabla\left(\chi(|z|>\bar{R}) j_{\bar{a}}\right) E_{K}^{t}(\bar{a}, x)\right\} p+O\left(\langle z\rangle^{-1}\right) .
$$

Using Lemma B.1 (1) we can estimate part of the second commutator by $e$ as follows:

$$
\begin{aligned}
i[-E \cdot x, A] & \geqq \chi(|z|>\bar{R}) \sum_{\bar{a} \in S(. \mathscr{C})} j_{\bar{a}}\left(e+\nabla r_{K}^{\bar{a}}(x) \cdot E\right) \\
& =\chi(|z|>\bar{R}) e+\chi(|z|>\bar{R}) \sum_{\bar{a} \in S(\mathscr{A})} j_{\bar{a}} \nabla r_{K}^{\bar{a}}(x) \cdot E .
\end{aligned}
$$

Keeping an application of Corollary 6.7 in mind we write using (S2) and (D4),

$$
i[-E \cdot x, A] \geqq e+\chi(|z|>\bar{R}) \sum_{\bar{a} \in S(\mathscr{A})} j_{\bar{a}} \sum_{1 \leqq k \leqq n(\bar{a})} K^{N-k} \frac{x \cdot E}{\left\langle x^{a_{k-1}}\right\rangle}+O\left(\langle z\rangle^{-1}\right) .
$$

Let $M(x)$ denote bounded matrix valued functions with bounded derivatives such that $M(x)^{t}=M(x)$. After substituting $x \cdot E=p^{2}-H+V$ into (B.6) we obtain

$$
i[H, A] \geqq e+p^{t}\left\{D_{1}+D_{2}\right\} p+T+i[V, A],
$$

where

$$
\begin{aligned}
D_{1} & =\chi(|z|>\bar{R}) \sum_{\bar{a} \in S(\mathscr{A})} \sum_{1 \leqq k \leqq n(\bar{a})} 2 \operatorname{Re}\left\{\vec{j}_{\bar{a}, k} E_{K}^{t}(\bar{a}, x)\right\} \\
D_{2} & =\chi(|z|>\bar{R}) \sum_{\bar{a} \in S(\mathscr{A})} \sum_{1 \leqq k \leqq n(\bar{a})} K^{N-k} j_{\bar{a}}\left\langle x^{a_{k-1}}\right\rangle^{-1} I, \\
T & =p^{t} \chi(|z|<2 \bar{R}) M(x) p+\sum_{\bar{a} \in S(\mathscr{A})} \sum_{1 \leqq k \leqq n(\bar{a})} T_{\bar{a}, k}+O\left(\langle z\rangle^{-1}\right)
\end{aligned}
$$


and

$$
T_{\bar{a}, k}=K^{N-k} \operatorname{Re}\left\{\left[\chi(|z|>\bar{R}) j_{\bar{a}}\left\langle x^{a_{k-1}}\right\rangle^{-1}, p\right] \cdot p+\chi(|z|>\bar{R}) j_{\bar{a}}\left\langle x^{a_{k-1}}\right\rangle^{-1}(V-H)\right\} .
$$

By summing over equivalence classes in $S_{k}(\mathscr{A})$ and applying Lemma B.1 (2-3) we obtain

$$
\begin{gathered}
D_{1}=\chi(|z|>\bar{R}) \sum_{k=1}^{N-1} \sum_{[\bar{a}] \in S_{k}(\mathscr{C}) / \sim} \sum_{\bar{b} \in[\bar{a}]} 2 \operatorname{Re}\left\{\vec{j}_{\bar{b}, k}\left(\sum_{k<l \leqq n(\bar{b})} K^{N-l}\left(\nabla r^{b_{l-1}}\right)^{t}+E(\bar{b})^{t}\right)\right\}, \\
D_{2}=\chi(|z|>\bar{R}) \sum_{k=1}^{N-1} \sum_{[\bar{a}] \in S_{k}(\mathscr{C}) / \sim} K^{N-k} j_{a_{1}}^{a_{0}} \ldots j_{a_{k-1}}^{a_{k-2}}\left\langle x^{\left.a_{k-1}\right\rangle^{-1} I .}\right.
\end{gathered}
$$

Adding $D_{1}$ and $D_{2}$ yields

$$
\begin{aligned}
D_{1}+D_{2}= & \chi(|z|>\bar{R}) \sum_{k=1}^{N-1} \sum_{[\bar{a}] \in S_{k}(\mathscr{A}) / \sim} j_{a_{1}}^{a_{0}} \ldots j_{a_{k-1}}^{a_{h-2}}\left\langle x^{a_{k-1}}\right\rangle^{-1} \\
& \times K^{N-1-k}\left(M_{k}^{[\bar{a}]}(x, K)+K I\right)
\end{aligned}
$$

where $K \rightarrow M_{k}^{[\bar{a}]}(\cdot, K)$ is a bounded map from $[1, \infty)$ to the bounded selfadjoint matrix valued functions on $X$ and $I$ is the identity. Thus by choosing a $K \geqq 1$ satisfying

$$
K \geqq \max _{\substack{[\bar{a}] \in S_{k}(\cdot, \mathcal{\prime}) / \sim \\ 1 \leqq h \leqq N-1}} \sup _{\tilde{K} \geqq 1} \sup _{x \in X}\left\|M_{k}^{[\bar{a}]}(x, \tilde{K})\right\|,
$$

we obtain

$$
D_{1}+D_{2} \geqq 0 \text {. }
$$

Using Proposition 6.4 and (B.3-4) we can write $T$ as

$$
T=\operatorname{Re}\left\{O\left(\langle z\rangle^{-1}\right) S\right\},
$$

where $S$ is $H$-bounded. Using Corollary 6.7 (cf. Proposition 3.7) with $\Pi=|\omega\rangle\langle\omega|$ we can find an $\varepsilon>0$ such that the following inequality holds for all $\lambda \in \mathscr{K}$ :

$$
\eta_{\varepsilon}(H-\lambda) T \eta_{\varepsilon}(H-\lambda) \geqq-\frac{e}{4} \eta_{\varepsilon}(H-\lambda)^{2} .
$$

Combining Lemma B.3 (2) and (B.7-9) we conclude that the proposition holds with the $\varepsilon$ and $K$ as introduced above.

Denote by $\operatorname{ad}_{A}$ the operation of commuting with $A$. That is $\operatorname{ad}_{A}(H)=[H, A]$. By (B.5) and the fact that

$$
i[H, A]=p^{t}\left\{E^{\prime}(x)+E^{\prime}(x)^{t}\right\} p-\Delta(\nabla \cdot E(x))+E(x) \cdot E-E(x) \cdot \nabla W,
$$

one easily obtains

Proposition B.5. For $n \in\{1,2\}$ we have

$$
\operatorname{ad}_{A}^{n}(H)=v_{n}+\sum_{1 \leqq|x| \leqq 2}\langle z\rangle^{-1} v_{\alpha, n}(x) p^{\alpha},
$$

where $v_{n}$ and $v_{\alpha, n}$ are continuous and bounded functions. 
This proposition combined with the properties (B.3-4) implies the following corollary.

Corollary B.6. The operator $\operatorname{ad}_{A}^{n}(H)$ is $H$-bounded for $n \in\{1,2\}$.

\section{References}

[A] Agmon, S.: Private communication

[ABG] Amrein, W.O., Boutet de Monvel, A.M., Georgescu, V.: Notes on the N-body problem; Part I. Preprint Univ. de Geneve UGVA DPT 1988/11-598a

[AHS] Agmon, S., Herbst, I., Skibsted, E.: Perturbation of embedded eigenvalues in the generalized N-body problem. Commun. Math. Phys. 122, 411-438 (1989)

[AS] Abramowitz, M., Stegun, I.A. (eds.): Handbook of mathematical functions. AMS 55, Department of Commerce, National Bureau of Standards, 1964

[D] Derezinski, J.: Asymptotic completeness of long range N-body quantum systems. Ann. Math. 138, 427-476 (1993)

[FH] Froese, R., Herbst I.: Exponential bounds and absence of positive eigenvalues for N-body Schrödinger operators. Commun. Math. Phys. 87, 429-447 (1982)

[H1] Herbst, I.: Temporal exponential decay for the Stark effect in atoms. J. Funct. Anal. 48, 224-251 (1982)

[H2] Herbst, I.: Perturbation theory for the decay rate of eigenfunctions in the generalized N-body problem. Commun. Math. Phys. 158, 517-536 (1993)

[HMS2] Herbst, I., Møller, J.S., Skibsted, E.: Asymptotic completeness of N-body Stark Hamiltonians. To appear in Commun. Math. Phys.

[HS] Halmos, P.R., Sunder, V.S.: Bounded Integral Operators on $L^{2}$ spaces. Berlin Heidelberg New York: Springer, 1978

[IO] Iorio, R.J. Jr., O'Carroll, M.: Asymptotic completeness for multi-particle Schroedinger Hamiltonians with weak potentials. Commun. Math. Phys. 27, 137-145 (1972)

[K] Korotyaev, E.L.: On the scattering theory of several particles in an external electric field. Math. USSR Sb. 60, 177-196 (1988)

[M] Mourre, E.: Absence of singular continuous spectrum for certain selfadjoint operators. Commun. Math. Phys. 91, 391-408 (1981)

[PSS] Perry, P., Sigal, I.M., Simon, B.: Spectral analysis of N-body Schrödinger operators. Ann. Math. 114, 519-567 (1981)

[RS] Reed, M., Simon, B.: Analysis of operators. Methods of modern mathematical physics IV, New York: Academic Press, 1978

[Si] Sigal, I.M.: Stark effect in multielectron systems: Non-existence of bound states. Commun. Math. Phys. 122, 1-22 (1989)

[Sk] Skibsted, E.: Absolute spectral continuity for N-body Stark Hamiltonians. Ann. Inst. Henri Poincaré 61, 2, 223-243 (1994)

[T1] Tamura, H.: Spectral and scattering theory for 3-particle Hamiltonian with Stark effect: Asymptotic completeness. Osaka J. Math. 29, 135-159 (1992)

[T2] Tamura, H.: Spectral and Scattering Theory for 3-Particle Hamiltonian with Stark effect: Non-existence of Bound States and Resolvent Estimate. Preprint 1993

[T3] Tamura, H.: Spectral analysis for N-particle systems with Stark effect: Non-existence of bound states and principle of limiting absorption. Preprint 1993

[T4] Tamura, H.: Principle of Limiting Absorption for N-body Schrödinger Operators. Lett. Math. Phys. 17, 31-36 (1989) 